\title{
Low-Rank Riemannian Optimization for Graph-Based Clustering Applications
}

\author{
Ahmed Douik, Member, IEEE and Babak Hassibi, Member, IEEE
}

\begin{abstract}
With the abundance of data, machine learning applications engaged increased attention in the last decade. An attractive approach to robustify the statistical analysis is to preprocess the data through clustering. This paper develops a low-complexity Riemannian optimization framework for solving optimization problems on the set of positive semidefinite stochastic matrices. The low-complexity feature of the proposed algorithms stems from the factorization of the optimization variable $\mathbf{X}=\mathbf{Y} \mathbf{Y}^{\mathrm{T}}$ and deriving conditions on the number of columns of $\mathbf{Y}$ under which the factorization yields a satisfactory solution. The paper further investigates the embedded and quotient geometries of the resulting Riemannian manifolds. In particular, the paper explicitly derives the tangent space, Riemannian gradients and Hessians, and a retraction operator allowing the design of efficient first and second-order optimization methods for the graph-based clustering applications of interest. The numerical results reveal that the resulting algorithms present a clear complexity advantage as compared with state-of-the-art Euclidean and Riemannian approaches for graph clustering applications.
\end{abstract}

Index Terms-Riemannian manifolds, Low-rank factorization, graphbased clustering, convex and non-convex optimization.

\section{INTRODUCTION}

$\mathrm{M}$ ULTIPLE non-convex optimization problems can be approximated using convex relaxations, e.g., non-negative matrix factorization [2], [3], compressive sensing [4], and matrix completion [5], [6]. Despite their convexity, solving these relaxed problems can often be a computation bottleneck in large-scale applications. Moreover, numerous relaxation techniques require expanding the dimension of the problem and the search space. For example, the celebrated lifting technique transforms an $n$-dimensional vector problem into a semidefinite $n \times n$ matrix problem. Luckily, these relaxations share an intrinsic property that greatly simplifies the computation, namely, the optimal solution is lowrank.

This manuscript considers solving convex and nonconvex optimization problems over the set of positive semidefinite stochastic matrices. Such problems arise in multiple communications [7], [8], signal processing [9], [10], and machine learning applications, notably in graph clustering and community detection [11], [12], [13], [14], [15], [16]. The factorization of the $n \times n$ optimization variable $\mathbf{X}=\mathbf{Y} \mathbf{Y}^{\mathrm{T}}$, with $\mathbf{Y}$ being an $n \times p$ matrix, allows us to reduce the search space from its original

A part of this paper [1] appears in proc. of International Conference on Machine Learning (ICML' 2018), Stockholm, Sweden.

Ahmed Douik and Babak Hassibi are with the Department of Electrical Engineering, California Institute of Technology, Pasadena, CA 91125 USA (e-mail: \{ahmed.douik,hassibi\}@caltech.edu). $n^{2}$ dimension to $n p$, which is extremely attractive for $p \ll n$. However, while convenient, the factorization turns the problem into a non-convex optimization with non-isolated solutions. Indeed, consider any solution $\mathbf{Y}$ and an orthogonal $p \times p$ matrix $\mathbf{O}$, the matrix $\mathbf{Y O}$ also represents a solution, i.e., the solution is invariant by right multiplication by an orthogonal matrix.

Riemannian manifold optimization offers the required tools for designing specialized algorithms for solving the aforementioned problems with moderate complexity. In particular, Riemannian quotient manifolds allow to group all solutions YO into the same equivalence class and solve the optimization problem over these classes. While this fact might be irrelevant for firstorder methods, it can be crucial for the convergence of second-order algorithms, e.g., Newton's method [17]. Consider an optimization problem over the set of positive semidefinite stochastic matrices. This paper suggests factorizing the optimization variable $\mathbf{X}=\mathbf{Y} \mathbf{Y}^{\mathrm{T}}$, deriving conditions on $p$ under which the factorization yields a satisfactory solution, and investigating the first and second-order geometries of the resulting manifolds so as to take advantage of Riemannian optimization to design highly efficient specialized algorithms.

\subsection{Related Work}

Factorizing a low-rank matrix and casting the optimization problem into a non-convex form is well studied in the literature, particularly for solving semidefinite programs (SDPs). For example, the factorization $\mathbf{X}=$ $\mathrm{YY}^{\mathrm{T}}$ is suggested in [18] to solve the maximum cut problem. The authors in [19] exploit similar factorization to solve SDPs with fixed traces. More generally, [20] investigates low-rank factorization for solving SDPs in standard form. The authors study the equivalence between the original and reformulated problems and propose a method for choosing the rank of the factorization in such a way to reduce complexity while preserving the optimality of the solution. This manuscript proposes similar theoretical guarantees for positive semidefinite stochastic matrices.

Optimization algorithms on Riemannian manifolds date back to the 1970's and 1980's with the works of Luenberger [21] and Gabay [22]. However, such optimization methods remained impracticable as they require the derivation of the exponential map whose computation is, aside from some special cases, as hard 
as solving the original optimization problem [17]. By the introduction of retractions [23] that successfully approximate the exponential map up to a given order, the last decade witnessed a high interest in optimization on Riemannian manifolds. A series of papers [24], [25], [26] extended the popular unconstrained algorithms to the Riemannian manifold setting, e.g., conjugate gradient and trust region methods.

Taking advantage of both the low-rank factorization and the optimization over Riemannian manifolds, the authors in [27] propose a first-order Riemannian algorithm for solving optimization problems on the elliptope, i.e., positive semidefinite matrices with ones on the diagonal. The quotient manifold is investigated in [28] and an invariant metric that makes the manifold geodesically complete is derived. A simpler quotient structure is introduced in [29] to solve optimization problems with general trace constraints, including the elliptope and the spectahedron, by proposing a secondorder algorithm with guaranteed quadratic convergence. This manuscript follows a similar approach in the sense that a novel quotient structure is proposed to solve optimization problems in which the optimization variable is a positive semidefinite stochastic matrix. Among the potential applications of the proposed manifold, this manuscript focuses, in Section 6, on a particular graphbased clustering technique, whose theoretical study is available in [12], to demonstrate the numerical advantages of the proposed method.

\subsection{Contributions}

Consider a positive semidefinite stochastic matrix $\mathbf{X}$ and the factorization $\mathbf{X}=\mathbf{Y Y}^{\mathrm{T}}$. The contribution of this paper is two-fold, namely providing theoretical guarantees and designing optimization algorithms for the reformulated problem. The first part of the manuscript exploits the factorization to cast the problem as a nonconvex optimization with non-isolated solutions. The optimality conditions of the original problem and its nonconvex reformulation are derived and analyzed. Such a theoretical study provides conditions under which the solution to the reformulated problem coincides with that of the original optimization problem. In particular, the analysis allows deriving a general procedure for solving the original problem in which the rank of the factorization is increased sequentially until the derived optimality conditions are satisfied. The procedure is further explained and simulated in Subsection 6.3.

The second part of the manuscript focuses on proposing first and second-order algorithms for solving the reparametrized optimization problem using tools from the Riemannian optimization. The authors in [30] introduce the doubly stochastic manifold to represent doubly stochastic matrices, wherein a doubly stochastic matrix refers to a non-negative entry-wise matrix whose columns and rows sum to unity. This work extends the results mentioned above to positive semidefinite stochastic matrices and its quotient manifold. In particular, the study of the manifolds geometries allows deriving first and second-order algorithms with guaranteed linear and quadratic convergence, respectively. Numerical experiments on clustering tasks reveal that the proposed algorithms present a clear complexity advantage over state-of-the-art Euclidean methods, namely, convex programming, and Riemannian approaches on the original non-reparametrized space, i.e., the doubly stochastic manifold.

The quotient structure investigated in this paper is fundamentally distinct from the embedded structure proposed in [30] and requires a different set of tools. Indeed, by grouping all points within the same equivalence class and considering them as same object, the quotient manifold significantly reduces the dimension of the problem and thus is expected to outperform its embedded counterpart, in general. As the manuscript does not assume prior knowledge of differential geometry or Riemannian optimization methods, all definitions, concepts, and theorems are briefly introduced. Interested reader are referred to the following references for an introduction to differential geometry [31], abstract manifold [32], Riemannian manifolds [33], and Riemannian optimization methods [17].

The rest of this paper is organized as follows: Section 2 formulates the optimization problem of interest and derives conditions under which the non-convex reparametrization yields a satisfactory solution. Section 3 introduces the essential tools for optimization over Riemannian manifolds and their quotient structures. The geometries of the embedded and quotient manifolds of interest are investigated in Section 4. The resulting specialized optimization algorithms can be found in Section 5. Finally, before concluding in Section 7, Section 6 plots and discusses the numerical results.

\section{Optimization on the Set of Positive Semidefinite Stochastic Matrices}

This section presents the optimization problems of interest and exploits the factorization to reparametrize them. After introducing the required notations and terminology, the optimality conditions of the original problem and its non-convex reformulation are derived and analyzed. The analysis reveals an efficient method for selecting the optimization rank while retaining equivalence with the original optimization problem.

\subsection{Problem Formation}

Let $\mathbf{X} \in \mathbb{R}^{n \times n}$ be a real symmetric $n \times n$ matrix and define the objective function $g: \mathbb{R}^{n \times n} \rightarrow \mathbb{R}$. While most applications possess a convex objective function, such assumption is not required herein. However, the paper assumes that the objective function is smooth, i.e., at least differentiable for convex functions and at least 
twice differentiable for non-convex ones. This paper is interested in solving the following problem

$$
\begin{array}{rl}
\min _{\mathbf{X} \in \mathbb{R}^{n \times n}} & g(\mathbf{X}) \\
\text { s.t. } & \mathbf{X}_{i j} \geq 0,1 \leq i, j \leq n \\
& \sum_{j=1}^{n} \mathbf{X}_{i j}=1,1 \leq i \leq n, \\
& \mathbf{X} \succeq \mathbf{0},
\end{array}
$$

wherein constraint (1b) underlines that the matrix is element-wise positive, constraint (1c) corresponds to the fact that the matrix is stochastic, and constraint (1d) insists that the matrix is positive semidefinite (PSD), i.e., the matrix is symmetric $\mathbf{X}=\mathbf{X}^{\mathrm{T}}$ and $\mathbf{v}^{\mathrm{T}} \mathbf{X} \mathbf{v} \geq 0$ for any vector $\mathbf{v} \in \mathbb{R}^{n}$. Notice that the combination of being symmetric, or PSD, and stochastic turns the matrix into a doubly stochastic one with all columns and rows summing to unity.

The optimization problem (1) requires searching for a solution in a space of dimension $\frac{n(n+1)}{2}=\mathcal{O}\left(n^{2}\right)$ which can be intractable for large-scale problems, e.g., community detection with millions of individuals. Nevertheless, as stated earlier, the applications of interest in this paper share the intrinsic property that the optimal solution has a much smaller rank than the ambient dimension. Such structure can be used to reduce by and large the computation complexity of both the optimization problem and the involved matrix operations, e.g., eigenvalue decomposition, Cholesky factorization.

The rest of the paper assumes that the optimal solution $\mathbf{X}^{*}$ has a rank $r$. If such rank is known a priori, one may directly use the appropriate Riemannian geometry introduced in Section 3. However, in most applications, such rank is unknown in which case one needs to increase the size of the model $p$ until the optimality conditions derived in the rest of this section are satisfied. The steps of the general approach for unknown rank $r$ can be found in Section 5 .

\subsection{Notation and Terminology}

Matrices and vectors are denoted with bold upper and lower characters, respectively, e.g., $\mathbf{X}$ and $\mathbf{v}$. The identity matrix is represented by the symbol $\mathbf{I}$. The all ones and all zeros vectors of length $n$ are denoted by $\mathbf{1}_{n}$ and $\mathbf{0}_{n}$, respectively. The index $n$ in $\mathbf{1}_{n}$ may be omitted if the dimension is clear from the context. The transpose, the inverse, and the $(i, j)$-th element of a matrix $\mathbf{X}$ are denoted by $\mathbf{X}^{\mathrm{T}}, \mathbf{X}^{-1}$, and $\mathbf{X}_{i j}$, respectively. A nonnegative semidefinite and an element-wise nonnegative matrix $\mathbf{X}$ are denoted by $\mathbf{X} \succeq \mathbf{0}$ and $\mathbf{X} \geq \mathbf{0}$, respectively.

Let the notation $\operatorname{Tr}($.$) refer to the trace operator and$ $\langle\mathbf{X}, \mathbf{Y}\rangle=\operatorname{Tr}\left(\mathbf{Y}^{\mathrm{T}} \mathbf{X}\right)$ to the Frobenius inner product of matrices $\mathbf{X}$ and $\mathbf{Y}$ on the space $\mathbb{R}^{n \times n}$. The Frobenius norm of a matrix $\mathbf{X}$ is defined as $\|\mathbf{X}\|_{F}=\sqrt{\operatorname{Tr}\left(\mathbf{X}^{\mathrm{T}} \mathbf{X}\right)}$.

Given two matrices, the element-wise product, a.k.a., the Hadamard product, is denoted by the symbol $\odot$, i.e., $(\mathbf{X} \odot \mathbf{Y})_{i j}=\mathbf{X}_{i j} \mathbf{Y}_{i j}$. Let $\mathcal{S}^{n}$ and $\mathcal{S}_{\text {skew }}^{n}$ be the set of symmetric and skew-symmetric matrices, respectively. A full rank $n \times p$ matrix $\mathbf{Y}$ is an element of the set $\mathbb{R}_{*}^{n \times p}$. The set $\mathcal{O}^{p}=\left\{\mathbf{O} \in \mathbb{R}_{*}^{p \times p} \mid \mathbf{O O}^{\mathrm{T}}=\mathbf{O}^{\mathrm{T}} \mathbf{O}=\mathbf{I}\right\}$ represents orthogonal matrices.

Consider a smooth function $f: \mathcal{E} \rightarrow \mathbb{R}$ from some Euclidean space $\mathcal{E}$. The directional derivative of $f(\mathbf{X})$ in the direction $\mathbf{Z} \in \mathcal{E}$ is defined as follows

$$
\mathrm{D}(f(\mathbf{X}))[\mathbf{Z}]=\lim _{t \rightarrow 0} \frac{f(\mathbf{X}+t \mathbf{Z})-f(\mathbf{X})}{t} .
$$

The operator $\mathbf{D}(f(\mathbf{X}))$ which associate to each $\mathbf{Z}$ the directional derivative $\mathrm{D}(f(\mathbf{X}))[\mathbf{Z}]$ is referred to as the indefinite directional derivative of $f$ at $\mathbf{X}$. The Euclidean gradient ${ }^{1}$ of $f(\mathbf{X})$ is defined as the unique matrix $\operatorname{Grad}(f(\mathbf{X}))$ satisfying

$$
\langle\operatorname{Grad}(f(\mathbf{X})), \mathbf{Z}\rangle=\mathrm{D}(f(\mathbf{X}))[\mathbf{Z}], \forall \mathbf{Z} \in \mathcal{E} .
$$

For functions of multiple variables, the variable for which the gradient is taken is explicitly written as a subscript, e.g., $\operatorname{Grad}_{\mathbf{X}}(f(\mathbf{X}, \mathbf{Y}))$ represents the gradient of the function with respect to the variable $\mathbf{X}$. Let Hess $(f(\mathbf{X}))[\mathbf{Z}]$ denote the Euclidean Hessian of $f(\mathbf{X})$ in the direction $\mathbf{Z}$ defined as the directional derivative of the gradient in that direction, i.e.,

$$
\operatorname{Hess}(f(\mathbf{X}))[\mathbf{Z}]=\mathrm{D}(\operatorname{Grad}(f(\mathbf{X})))[\mathbf{Z}] .
$$

\subsection{The Non-Convex Reformulation and Optimality Conditions}

As stated previously, solving problem (1) requires searching for a solution in an $\frac{n(n+1)}{2}$-dimensional space. To alleviate such computation burden, this section proposes using the low-rank decomposition $\mathbf{X}=\mathbf{Y Y}^{\mathrm{T}}$ wherein $\mathbf{Y} \in \mathbb{R}^{n \times p}$. Hence, the reformulated optimization problem has the form

$$
\begin{array}{rl}
\min _{\mathbf{Y} \in \mathbb{R}^{n \times p}} & g\left(\mathbf{Y Y}^{\mathrm{T}}\right) \\
\text { s.t. } & \mathbf{Y} \mathbf{Y}^{\mathrm{T}} \geq \mathbf{0} \\
& \mathbf{Y} \mathbf{Y}^{\mathrm{T}} \mathbf{1}=\mathbf{1} .
\end{array}
$$

Notice that the original PSD constraint (1d) is implicitly assumed in the structure $\mathbf{Y Y}^{\mathrm{T}}$ which is always a PSD matrix. The reparametrized problem requires searching for a solution in an $n p \ll \frac{n(n+1)}{2}$ dimensional space. However, even under the assumption that the optimization problem (1) is convex, the reformulated problem (2) is non-convex.

Ideally, the decomposition rank $p$ would coincide with the actual rank $r$ of the solution $\mathbf{X}^{*}$. However, such rank being unknown in most applications, the rest of this section derives conditions under which the reformulated problem and the original one are equivalent. In other words, this section derives conditions under which an extreme point $\mathbf{Y}$ of (2) corresponds to an extreme point $\mathbf{X}=\mathbf{Y Y}^{\mathrm{T}}$ of (1). To this end, first and second order optimality conditions are derived. These results extend the findings of [20] to non-convex functions with inequality constraints.

1. As per the notation convention in the Riemannian geometry literature, the symbol $\nabla$ is reserved for the Riemannian connection on the manifold as introduced in Section 3. 
The optimality condition of problem (1) are obtained from the first-order Karush-Kuhn-Tucker (KKT) conditions [34]. To simplify the analysis, the optimization problem is rewritten in a symmetric form as follows

$$
\begin{array}{rl}
\min _{\mathbf{X} \in \mathbb{R}^{n \times n}} & g(\mathbf{X}) \\
\text { s.t. } & \mathbf{X}+\mathbf{X}^{\mathrm{T}} \geq \mathbf{0} \\
& \left(\mathbf{X}+\mathbf{X}^{\mathrm{T}}\right) \mathbf{1}=\mathbf{2} \\
& \mathbf{X} \succeq \mathbf{0} .
\end{array}
$$

Let $\overline{\mathbf{\Psi}}, \sigma$ and $\mathbf{S}_{\mathbf{X}}$ be the dual variables corresponding to constraints (3b), (3c), and (3d), respectively. The Lagrangian $\mathcal{L}_{\mathbf{X}}\left(\mathbf{X}, \sigma, \mathbf{S}_{\mathbf{X}}, \overline{\mathbf{\Psi}}\right)$ can be written as

$$
\begin{aligned}
& \mathcal{L}_{\mathbf{X}}\left(\mathbf{X}, \sigma, \mathbf{S}_{\mathbf{X}}, \overline{\mathbf{\Psi}}\right)=g(\mathbf{X})+\left\langle\sigma,\left(\mathbf{X}+\mathbf{X}^{\mathrm{T}}\right) \mathbf{1}-\mathbf{2}\right\rangle \\
& \quad-\left\langle\bar{\Psi}, \mathbf{X}+\mathbf{X}^{\mathrm{T}}\right\rangle-\left\langle\mathbf{S}_{\mathbf{X}}, \mathbf{X}\right\rangle, \quad \overline{\mathbf{\Psi}} \geq \mathbf{0}, \mathbf{S}_{\mathbf{X}} \succeq \mathbf{0} .
\end{aligned}
$$

Differentiating the above Lagrangian with respect to the variable $\mathbf{X}$ gives $\operatorname{Grad}_{\mathbf{X}}(g(\mathbf{X}))+\sigma \mathbf{1}^{\mathrm{T}}+\mathbf{1} \sigma^{\mathrm{T}}-\mathbf{S}_{\mathbf{X}}-$ $\overline{\mathbf{\Psi}}-\overline{\mathbf{\Psi}}^{\mathrm{T}}=0$ with the corresponding positiveness and complementary slackness constraints $\mathbf{S}_{\mathbf{X}} \succeq \mathbf{0}, \overline{\mathbf{\Psi}} \geq \mathbf{0}$, $\mathbf{X} \odot \overline{\boldsymbol{\Psi}}=\mathbf{0}$, and $\mathbf{S}_{\mathbf{X}} \mathbf{X}=\mathbf{0}$. Let $\boldsymbol{\Psi}=\overline{\mathbf{\Psi}}+\overline{\boldsymbol{\Psi}}^{\mathrm{T}} \in \mathcal{S}^{n}$, the non-negativity constraints $\overline{\boldsymbol{\Psi}} \geq \mathbf{0}$ and $\boldsymbol{\Psi} \geq \mathbf{0}$ along with the complementary slackness conditions $\mathbf{X} \odot \overline{\mathbf{\Psi}}=\mathbf{0}$ and $\mathbf{X} \odot \mathbf{\Psi}=\mathbf{0}$ are equivalent. Finally, the uniqueness of the dual variables derives from the independence of the constraints [35].

Combining all the above, a solution $\mathbf{X} \in \mathbb{R}^{n \times n}$ is an extreme point of the optimization problem (1) if there exists the unique dual variables $\sigma \in \mathbb{R}^{n}, \mathbf{S}_{\mathbf{X}}, \Psi \in \mathcal{S}^{n}$ such that the following equations hold

$$
\begin{aligned}
\operatorname{Grad}_{\mathbf{X}}(g(\mathbf{X}))+\sigma \mathbf{1}^{\mathrm{T}} & +\mathbf{1} \sigma^{\mathrm{T}}=\mathbf{S}_{\mathbf{X}}+\mathbf{\Psi} \\
\mathbf{X} & \succeq \mathbf{0} \\
\mathbf{X} & \geq \mathbf{0} \\
\mathbf{X} \mathbf{1} & =\mathbf{1} \\
\mathbf{S}_{\mathbf{X}} & \succeq \mathbf{0} \\
\mathbf{\Psi} & \geq \mathbf{0} \\
\mathbf{X} \odot \mathbf{\Psi} & =\mathbf{0} \\
\mathbf{S}_{\mathbf{X}} \mathbf{X} & =\mathbf{0},
\end{aligned}
$$

with constraint (4a) translating the fact $\left(\mathbf{X}, \sigma, \mathbf{S}_{\mathbf{X}}, \mathbf{\Psi}\right)$ is a saddle point for the Lagrangian, (4b)-(4d) stating that the solution is a feasible point, (4e) and (4f) representing the positiveness of the dual variables and $(4 \mathrm{~g})$ and $(4 \mathrm{~h})$ expressing the complementary slackness.

A similar analysis on the reparametrized optimization problem (2) allows to conclude that a solution $\mathbf{Y} \in \mathbb{R}^{n \times p}$ is an extreme point of (2) if there exists the unique dual variables $\lambda \in \mathbb{R}^{n}$ and $\boldsymbol{\Phi} \in \mathcal{S}^{n}$ satisfying the following

$$
\begin{aligned}
\left(\operatorname{Grad}_{\mathbf{X}}\left(g\left(\mathbf{Y} \mathbf{Y}^{\mathrm{T}}\right)\right)+\lambda \mathbf{1}^{\mathrm{T}}\right. & \left.+\mathbf{1} \lambda^{\mathrm{T}}-\mathbf{\Phi}\right) \mathbf{Y}=\mathbf{0} \\
\mathbf{Y} \mathbf{Y}^{\mathrm{T}} & \geq \mathbf{0} \\
\mathbf{Y} \mathbf{Y}^{\mathrm{T}} \mathbf{1} & =\mathbf{1} \\
\mathbf{\Phi} & \geq \mathbf{0} \\
\mathbf{Y Y} \mathbf{Y}^{\mathrm{T}} \odot \mathbf{\Phi} & =\mathbf{0},
\end{aligned}
$$

with constraint (5a) translating the fact $(\mathbf{Y}, \lambda, \mathbf{\Phi})$ is a saddle point for the Lagrangian, $(5 b)$ and $(5 c)$ stating that the solution is a feasible point, $(5 \mathrm{~d})$ representing the positiveness of the dual variable and (5e) expressing the complementary slackness.

Theorem 1. An extreme point $\mathbf{Y}$ of the optimization problem (2) produces an extreme point $\mathbf{X}=\mathbf{Y} \mathbf{Y}^{\mathrm{T}}$ of the problem (1) if and only if the following holds

$$
\operatorname{Grad}_{\mathbf{X}}\left(g\left(\mathbf{Y} \mathbf{Y}^{\mathrm{T}}\right)\right)+\lambda \mathbf{1}^{\mathrm{T}}+\mathbf{1} \lambda^{\mathrm{T}}-\mathbf{\Phi}=\mathbf{S}_{\mathbf{Y}} \succeq \mathbf{0},
$$

wherein $\lambda$ and $\mathbf{\Phi}$ are the dual variable associated with $\mathbf{Y}$.

Proof: Let $\mathbf{Y}$ be an extreme point of (2). Following the above analysis, there exists the dual variables $\sigma$ and $\boldsymbol{\Phi}$ satisfying the conditions (5)-(5e). Let $\mathbf{S}_{\mathbf{Y}}$ be defined as $\mathbf{S}_{\mathbf{Y}}=\operatorname{Grad}_{\mathbf{X}}\left(g\left(\mathbf{Y} \mathbf{Y}^{\mathrm{T}}\right)\right)+\lambda \mathbf{1}^{\mathrm{T}}+\mathbf{1} \lambda^{\mathrm{T}}-\mathbf{\Phi}$. The sufficient part of the proof is straightforward by considering the identification $\mathbf{X}=\mathbf{Y Y}^{\mathrm{T}}, \sigma=\lambda, \mathbf{S}_{\mathbf{X}}=\mathbf{S}_{\mathbf{Y}}$, and $\boldsymbol{\Psi}=\boldsymbol{\Phi}$. Indeed, one can clearly see that the optimality conditions (5)-(5e) with the positiveness of $\mathbf{S}_{\mathbf{Y}} \succeq \mathbf{0}$ result in the optimality conditions (4b)-(4h). Furthermore, by definition of $\mathbf{S}_{\mathbf{Y}}$, the constraint (4a) is satisfied which concludes by virtue of the optimality conditions in (4) that the point is an extreme point of the optimization problem (1).

Conversely, assume that $\mathbf{X}=\mathbf{Y} \mathbf{Y}^{\mathrm{T}}$ is an extreme point of (1) for some dual variables $\sigma, \mathbf{S}_{\mathbf{X}}$, and $\boldsymbol{\Psi}$. In order to show that $\mathbf{S}_{\mathbf{Y}} \succeq \mathbf{0}$, we exploit the uniqueness of the dual variables to establish the equality $\sigma=\lambda$ and $\Psi=\boldsymbol{\Phi}$. From the optimality conditions in (4), by multiplying (4a) by $\mathrm{X} 1$ and using the identity in (4d) and (4h), we obtain the following equation

$$
\left(\sigma \mathbf{1}^{\mathrm{T}}+\mathbf{1} \sigma^{\mathrm{T}}\right) \mathbf{1}=\left(\mathbf{\Psi}-\operatorname{Grad}_{\mathbf{X}}\left(g\left(\mathbf{Y} \mathbf{Y}^{\mathrm{T}}\right)\right)\right) \mathbf{1} .
$$

Solving for $\sigma$ in the above expression gives

$$
\begin{aligned}
\left(n \mathbf{I}+\mathbf{1 1}^{\mathrm{T}}\right) \sigma & =\left(\mathbf{\Psi}-\operatorname{Grad}_{\mathbf{X}}\left(g\left(\mathbf{Y} \mathbf{Y}^{\mathrm{T}}\right)\right)\right) \mathbf{1} \\
\sigma & =\frac{1}{n}\left(\mathbf{I}-\frac{1}{2 n} \mathbf{1 1}^{\mathrm{T}}\right)\left(\mathbf{\Psi}-\operatorname{Grad}_{\mathbf{X}}\left(g\left(\mathbf{Y} \mathbf{Y}^{\mathrm{T}}\right)\right)\right) \mathbf{1} .
\end{aligned}
$$

Using the optimality conditions in (5) and combining the equations (5a) and (5c) yields the following equation for $\lambda$

$$
\lambda=\frac{1}{n}\left(\mathbf{I}-\frac{1}{2 n} \mathbf{1 1}^{\mathrm{T}}\right)\left(\mathbf{\Phi}-\operatorname{Grad}_{\mathbf{X}}\left(g\left(\mathbf{Y} \mathbf{Y}^{\mathrm{T}}\right)\right)\right) \mathbf{1} .
$$

Exploiting the uniqueness of the variables, we conclude that $\sigma=\lambda$ and $\mathbf{\Phi}=\mathbf{\Psi}$. Finally, using the definition of $\mathbf{S}_{\mathbf{Y}}=\operatorname{Grad}_{\mathbf{X}}\left(g\left(\mathbf{Y} \mathbf{Y}^{\mathrm{T}}\right)\right)+\lambda \mathbf{1}^{\mathrm{T}}+\mathbf{1} \lambda^{\mathrm{T}}-\mathbf{\Phi}$ with $\lambda=\sigma$ and $\boldsymbol{\Psi}=\boldsymbol{\Phi}$ gives $\mathbf{S}_{\mathbf{X}}=\mathbf{S}_{\mathbf{Y}}$. Therefore, the optimality condition (4e) results in $\mathbf{S}_{\mathbf{Y}} \succeq \mathbf{0}$ which concludes the proof.

The above first-order analysis and result in Theorem 1 are sufficient to declare optimality for optimization problems with a convex objective function $g$. For non-convex functions, the following theorem investigates the secondorder optimality conditions.

Theorem 2. A sufficient condition for an extreme point $\mathbf{Y} \in$ $\mathbb{R}^{n \times p}$ of (2) to yield an extreme point $\mathbf{X}=\mathbf{Y} \mathbf{Y}^{\mathrm{T}}$ of (1) is that $\operatorname{Rank}(\mathbf{Y})<p$.

Proof: The proof of this theorem relies on the secondorder KKT conditions of the optimization problem (2). These conditions, combined with the rank deficiency assumption, allow showing that $\mathbf{S}_{\mathbf{Y}}$ is positive semidef- 
inite which by virtue of Theorem 1 concludes that $\mathbf{X}$ is an extreme point of (1).

Proposition 1. Let $(\mathbf{Y}, \lambda, \mathbf{\Phi})$ satisfy the first-order KKT conditions in (5) and let $\mathbf{S}_{\mathbf{Y}}=\operatorname{Grad}_{\mathbf{X}}\left(g\left(\mathbf{Y Y}^{\mathrm{T}}\right)\right)+\lambda \mathbf{1}^{\mathrm{T}}+$ $\mathbf{1} \lambda^{\mathrm{T}}-\boldsymbol{\Phi}$. For all matrices $\mathbf{Z} \in \mathbb{R}^{n \times p}$ satisfying $\mathbf{Y} \mathbf{Z}^{\mathrm{T}}=\mathbf{0}$, it holds that $\operatorname{Tr}\left(\mathbf{Z}^{\mathrm{T}} \mathbf{S}_{\mathbf{Y}} \mathbf{Z}\right) \geq 0$.

Proof: Let the optimization problem (2) be rewritten in a more systematic form as

$$
\begin{aligned}
\min _{\mathbf{Y} \in \mathbb{R}^{n \times p}} & \frac{1}{2} g\left(\mathbf{Y} \mathbf{Y}^{\mathrm{T}}\right) \\
\text { s.t. } & \operatorname{Tr}\left(\mathbf{E}_{i j}^{\mathrm{T}} \mathbf{Y} \mathbf{Y}^{\mathrm{T}}\right) \geq 0,1 \leq i, j \leq n \\
& \operatorname{Tr}\left(\mathbf{E}_{i}^{\mathrm{T}} \mathbf{Y} \mathbf{Y}^{\mathrm{T}}\right)=1,1 \leq i \leq n,
\end{aligned}
$$

wherein $\mathbf{E}_{i j}$ is the matrix with 1 on the $(i, j)$-th entry and zeros elsewhere and $\mathbf{E}_{i}$ is the matrix with ones on the $i$-th column and zeros elsewhere. The analysis of the second order conditions of the above problem reveal the inequality

$$
\operatorname{Tr}\left(\mathbf{Z}^{\mathrm{T}} \operatorname{Hess}_{\mathbf{Y}}\left(\operatorname{Grad}_{\mathbf{Y}} \mathcal{L}_{\mathbf{Y}}(\mathbf{Y}, \lambda, \boldsymbol{\Phi})[\mathbf{Z}]\right)\right) \geq 0,
$$

for all matrix $\mathbf{Z} \in \mathbb{R}^{n \times p}$ satisfying the following

$$
\begin{aligned}
& \operatorname{Tr}\left(\mathbf{Z}^{\mathrm{T}}\left(\mathbf{E}_{i j}+\mathbf{E}_{j i}\right) \mathbf{Y}\right)=0,1 \leq i, j \leq n, \boldsymbol{\Phi}_{i j}>0 \\
& \operatorname{Tr}\left(\mathbf{Z}^{\mathrm{T}}\left(\mathbf{E}_{i j}+\mathbf{E}_{j i}\right) \mathbf{Y}\right)=0,1 \leq i, j \leq n, \boldsymbol{\Phi}_{i j}=0 \\
& \operatorname{Tr}\left(\mathbf{Z}^{\mathrm{T}}\left(\mathbf{E}_{i}+\mathbf{E}_{i}^{\mathrm{T}}\right) \mathbf{Y}\right) \geq 0,1 \leq i \leq n .
\end{aligned}
$$

In particular, note that the inequality in (7) is achieved for any matrix $\mathbf{Z} \in \mathbb{R}^{n \times p}$ satisfying $\mathbf{Y Z}^{\mathrm{T}}=\mathbf{0}$. Indeed, the condition $\mathbf{Y Z}^{\mathrm{T}}=\mathbf{0}$ implies the three conditions in (8), (9), and (10). Furthermore, noting that $\operatorname{Grad}_{\mathbf{Y}} \mathcal{L}_{\mathbf{Y}}(\mathbf{Y}, \lambda, \boldsymbol{\Phi})=\mathbf{S}_{\mathbf{Y}} \mathbf{Y}$ and under the constraint $\mathbf{Y Z}^{\mathrm{T}}=\mathbf{0}$, the left-hand side of (7) simplifies as follows

$$
\begin{aligned}
& \operatorname{Tr}\left(\mathbf{Z}^{\mathrm{T}} \operatorname{Hess}_{\mathbf{Y}}\left(\operatorname{Grad}_{\mathbf{Y}} \mathcal{L}_{\mathbf{Y}}(\mathbf{Y}, \lambda, \boldsymbol{\Phi})[\mathbf{Z}]\right)\right) \\
& \quad=\operatorname{Tr}\left(\mathbf{Z}^{\mathrm{T}} \mathrm{D}\left(\mathbf{S}_{\mathbf{Y}}\right)[\mathbf{Z}] \mathbf{Y}\right)+\operatorname{Tr}\left(\mathbf{Z}^{\mathrm{T}} \mathbf{S}_{\mathbf{Y}} \mathbf{Z}\right) \\
& \quad=\operatorname{Tr}\left(\mathbf{Z}^{\mathrm{T}} \mathbf{S}_{\mathbf{Y}} \mathbf{Z}\right),
\end{aligned}
$$

wherein the last equality is obtained from the fact that $\operatorname{Tr}\left(\mathbf{Z}^{\mathrm{T}} \mathrm{D}\left(\mathbf{S}_{\mathbf{Y}}\right)[\mathbf{Z}] \mathbf{Y}\right)=\operatorname{Tr}\left(\mathbf{D}\left(\mathbf{S}_{\mathbf{Y}}\right)[\mathbf{Z}] \mathbf{Y} \mathbf{Z}^{\mathrm{T}}\right)=0$. Therefore, we obtain $\operatorname{Tr}\left(\mathbf{Z}^{\mathrm{T}} \mathbf{S}_{\mathbf{Y}} \mathbf{Z}\right) \geq 0$ for all $\mathbf{Z}$ such that $\mathbf{Y} \mathbf{Z}^{\mathrm{T}}=\mathbf{0}$

The rest of the proof uses the result of Proposition 1 and shows that for a rank deficient solution $\mathbf{Y}$, the inequality $\operatorname{Tr}\left(\mathbf{Z}^{\mathrm{T}} \mathbf{S}_{\mathbf{Y}} \mathbf{Z}\right) \geq 0$ implies the positiveness of $\mathbf{S}_{\mathbf{Y}} \succeq \mathbf{0}$ which by virtue of Theorem 1 results in $\mathbf{X}$ being an extreme point of (1). The above mentioned steps are omitted as they mirrors the steps used in proving Theorem 7 in [29].

\section{Optimization on RiemanNian Embedded AND QUOTIENT MANIFOLDS}

The fundamental idea of optimization algorithms on manifolds is to locally approximate the manifold by a linear space known as the tangent space. Afterwards, unconstrained optimization is performed on that tangent space. In particular, a descent direction is computed by deriving the Riemannian gradient. Finally, the point on the tangent space is projected to the manifold using a retraction.
TABLE 1

Embedded and Quotient Manifolds Notations

\begin{tabular}{|c|c|}
\hline Variable & Definition \\
\hline \hline $\mathcal{M}, \overline{\mathcal{M}}$ & Embedded manifold and its quotient \\
\hline $\mathbf{Y}$ and $\overline{\mathbf{Y}}=[\mathbf{Y}]$ & A point on $\mathcal{M}$ and its class on $\overline{\mathcal{M}}$ \\
\hline \hline$\xi_{\mathbf{Y}} \in \mathcal{T}_{\mathbf{Y}} \mathcal{M}$ & A point on the tangent space of $\mathcal{M}$ \\
\hline $\mathcal{H}_{\mathbf{Y}} \mathcal{M}, \mathcal{V}_{\mathbf{Y}} \mathcal{M}$ & Horizontal and vertical spaces at $\mathbf{Y}$ \\
\hline $\mathcal{P}_{\mathbf{Y}}$ & Orthogonal projection onto $\mathcal{T}_{\mathbf{Y}} \mathcal{M}$ \\
\hline $\mathcal{P}_{\mathbf{Y}}^{\mathcal{H}}$ & Orthogonal projection onto $\mathcal{H}_{\mathbf{Y}} \mathcal{M}$ \\
\hline $\mathcal{P}_{\mathbf{Y}}^{\mathcal{V}}$ & Orthogonal projection onto $\mathcal{V}_{\mathbf{V}} \mathcal{M}$ \\
\hline$\xi_{\overline{\mathbf{Y}}} \in \mathcal{T}_{\overline{\mathbf{Y}}} \overline{\mathcal{M}}$ & A point on the tangent of $\overline{\mathcal{M}}$ \\
\hline $\bar{\xi}_{\mathbf{Y}} \in \mathcal{H}_{\mathbf{Y}} \mathcal{M}$ & Horizontal lift of $\xi_{\overline{\mathbf{Y}}}$ at $\mathbf{Y} \in \pi^{-1}(\overline{\mathbf{Y}})$ \\
\hline \hline $\operatorname{Grad}_{f}(\mathbf{Y})$ & Euclidean Gradient at $\mathbf{Y}$ \\
\hline Hess $f(\mathbf{Y})\left[\xi_{\mathbf{Y}}\right]$ & Euclidean Hessian at $\mathbf{Y}$ and $\xi \mathbf{Y}$ \\
\hline $\operatorname{grad} f(\mathbf{Y})$ & Riemannian Gradient at $\mathbf{Y}$ \\
\hline hess $f(\mathbf{Y})\left[\xi_{\mathbf{Y}}\right]$ & Riemannian Hessian at $\mathbf{Y}$ and $\xi \mathbf{Y}$ \\
\hline $\operatorname{grad} \bar{f}(\mathbf{Y})$ & Lift of grad $\bar{f}(\overline{\mathbf{Y}})$ at $\mathbf{Y} \in \pi^{-1}(\overline{\mathbf{Y}})$ \\
\hline hess $\bar{f}(\mathbf{Y})\left[\xi_{\mathbf{Y}}\right]$ & Lift of hess $\bar{f}(\overline{\mathbf{Y}})\left[\xi_{\mathbf{Y}}\right]$ at $\mathbf{Y} \in \pi^{-1}(\overline{\mathbf{Y}})$ \\
\hline \hline $\operatorname{R}_{\mathbf{Y}}\left(\xi_{\mathbf{Y}}\right)$ & Retraction of $\xi_{\mathbf{Y}}$ at $\mathbf{Y} \in \mathcal{M}$ \\
\hline $\bar{R}_{\overline{\mathbf{Y}}}\left(\xi_{\overline{\mathbf{Y}}}\right)$ & Retraction of lift $\overline{\xi_{\mathbf{Y}}}$ at $\mathbf{Y} \in \pi^{-1}(\overline{\mathbf{Y}})$ \\
\hline
\end{tabular}

This section defines the above relevant concepts from differential and Riemannian geometry. In particular, Subsection 3.1 introduces the essential tools for optimization over Riemannian embedded manifolds. Subsection 3.2 provides the tools for optimization over quotient Riemannian manifolds. The definitions and notations used herein can be found in the book [17]. In the rest of the manuscript, variables relative to the quotient manifold, e.g., equivalence classes, are denoted by overline characters and their representatives in the embedded manifold are represented without the overline. For convenience, Table 1 summarizes all notations used in this paper.

\subsection{Manifold Optimization: Definitions and Notation}

Let $\mathcal{M}$ be a matrix manifold embedded in the set of matrices $\mathbb{R}^{n \times p}$, known as the embedding space. The manifold is said to have a dimension $d$, also known as the degrees of freedom, if there exists a mapping from the manifold to an open subset of $\mathbb{R}^{d}$. Given a point $\mathbf{Y}$ on the manifold, the tangent space $\mathcal{T}_{\mathbf{Y}} \mathcal{M}$ is a $d$-dimensional Euclidean space that approximates the manifold $\mathcal{M}$ at $\mathbf{Y}$. Such tangent space is generated by computing the speed at the origin of all curves in $\mathcal{M}$ going through and rooted at $\mathbf{Y}$.

Tangent spaces play a primary role in optimization methods over Riemannian manifolds as they allow to locally transform the curved manifold into a smooth vector space to which common unconstrained optimization techniques can be applied. However, one needs the notion of distance and length on these tangent spaces to apply optimization algorithms. Such a notion is provided by a bilinear, symmetric, positive, and smoothly varying form, known as the Riemannian metric. The restriction of the Riemannian metric to the tangent space 
$\mathcal{T}_{\mathbf{Y}} \mathcal{M}$ forms an inner-product denoted by $\langle., .\rangle_{\mathbf{Y}}$. In the rest of the manuscript, tangent vectors are denoted by Greek letters wherein the point on the manifold in which the tangent space is computed is given as a subscript. In particular, the norm of a tangent vector $\xi_{\mathbf{Y}} \in \mathcal{T}_{\mathbf{Y}} \mathcal{M}$ is denoted by the following

$$
\left\|\xi_{\mathbf{Y}}\right\|_{\mathbf{Y}}^{2}=\left\langle\xi_{\mathbf{Y}}, \xi_{\mathbf{Y}}\right\rangle_{\mathbf{Y}}, \forall \xi_{\mathbf{Y}} \in \mathcal{T}_{\mathbf{Y}} \mathcal{M}
$$

As stated earlier, this manuscript considers the induced Frobenius inner product as a Riemannian metric. Therefore, the first order derivative, i.e., the Riemannian gradient, is obtained by taking the component of the Euclidean gradient in the tangent space. Let $\mathcal{P}_{\mathbf{Y}}: \mathbb{R}^{n \times p} \rightarrow$ $\mathcal{T}_{\mathbf{Y}} \mathcal{M}$ denote the orthogonal projection from the ambient space to the tangent one, then the Riemannian gradient can be written as a function of the Euclidean one as follows

$$
\operatorname{grad} f(\mathbf{Y})=\mathcal{P}_{\mathbf{Y}}(\operatorname{Grad} f(\mathbf{Y})) \text {. }
$$

The second order derivative, known as the Riemannian Hessian, is obtained by taking covariant derivative of the Riemannian gradient. Such notion of covariant derivative of a tangent vector $\eta_{\mathbf{Y}}$ in the direction $\xi_{\mathbf{Y}}$ is provided by the operator $\nabla_{\xi_{\mathbf{Y}}} \eta_{\mathbf{Y}}$, called a Riemannian connection. Using the above definition, the Riemannian Hessian hess $g(\mathbf{Y})\left[\xi_{\mathbf{Y}}\right]$ at the point $\mathbf{Y} \in \mathcal{M}$ and acting on the tangent vector $\xi_{\mathbf{Y}}$, is defined as the operator that satisfies the following

$$
\text { hess } f(\mathbf{Y})\left[\xi_{\mathbf{Y}}\right]=\nabla_{\xi \mathbf{Y}} \operatorname{grad} f(\mathbf{Y}) \text {. }
$$

A canonical choice for the Riemannian connection is the Levi-Civita affine connection. As manifolds in this paper are embedded in a vector space with its canonical inner product, $\mathbb{R}^{n \times p}$, the Riemannian connection $\nabla_{\xi_{\mathbf{Y}}}$ reduces to a simple directional derivative followed by a projection onto the tangent space. Hence, the Riemannian Hessian can be expressed as

$$
\text { hess } f(\mathbf{Y})\left[\xi_{\mathbf{Y}}\right]=\mathcal{P}_{\mathbf{Y}}\left(\mathrm{D}(\operatorname{grad} f(\mathbf{Y}))\left[\xi_{\mathbf{Y}}\right]\right) \text {. }
$$

After choosing the descent direction using the derivative information, the step size is chosen according to Wolfe's conditions, i.e., the Armijo and curvature conditions [17]. Finally, the tangent vector is retracted to the manifold using the retraction operator $\mathrm{R}$ whose restriction $R_{Y}$ to the tangent space $\mathcal{T}_{\mathbf{Y}} \mathcal{M}$ satisfies the centering and local rigidity properties [17]. In other words, the retraction satisfies

$$
\begin{aligned}
\mathbf{R}_{\mathbf{Y}}(\mathbf{0}) & =\mathbf{Y} \\
\left.\frac{d \mathbf{R}_{\mathbf{Y}}(\tau \xi \mathbf{Y})}{d \tau}\right|_{\tau=0} & =\xi_{\mathbf{Y}} .
\end{aligned}
$$

\subsection{Optimization on Quotient Riemannian Manifolds}

Let $\sim$ be an equivalence relationship and define the set $\overline{\mathcal{M}}=\mathcal{M} / \sim$ as the quotient of the manifold $\mathcal{M}$ by $\sim$. Under some mild conditions on $\sim$, available in Appendix $\mathrm{A}$, the set $\overline{\mathcal{M}}$ admits a manifold structure. In other words, the quotient manifold $\overline{\mathcal{M}}$ groups all elements of $\mathcal{M}$ in the same equivalence class as a single point. Let $\pi$ be the natural projection that associates to each $\mathbf{Y} \in \mathcal{M}$ its equivalence class $\pi(\mathbf{Y})=[\mathbf{Y}]=\overline{\mathbf{Y}} \in \overline{\mathcal{M}}$.
These three notations for equivalence classes are used interchangeably in this paper.

Let $\langle., .\rangle_{\mathbf{Y}}$ be the Riemannian metric on the tangent space $\mathcal{T}_{\mathbf{Y}} \mathcal{M}$ of the embedding space $\mathcal{M}$. The quotient $\overline{\mathcal{M}}=\mathcal{M} / \sim$ admits a Riemmanian structure for the induced Riemannian metric if and only if the metric is compatible with the equivalence relationship $\sim$, i.e., it does not depend on the chosen representative of the equivalence class.

To express the compatibility of the metric, we first introduce the horizontal lift. For a point $\overline{\mathbf{Y}} \in \overline{\mathcal{M}}$, let $\xi_{\overline{\mathbf{Y}}} \in$ $\mathcal{T}_{\overline{\mathbf{Y}}} \overline{\mathcal{M}}$ be a tangent vector. In a similar manner that $\overline{\mathbf{Y}}$ can be represented by multiple $\mathbf{Y} \in \pi^{-1}(\overline{\mathbf{Y}})$, the tangent vector $\xi_{\overline{\mathbf{Y}}}$ can be represented by multiple predecessors for each $\mathbf{Y} \in \pi^{-1}(\overline{\mathbf{Y}})$. Indeed, fix $\mathbf{Y} \in \pi^{-1}(\overline{\mathbf{Y}})$, then any tangent vector $\xi_{\mathbf{Y}} \in \mathcal{T}_{\mathbf{Y}} \mathcal{M}$ satisfying $\mathrm{D}(\pi(\mathbf{Y}))\left[\xi_{\mathbf{Y}}\right]=$ $\xi_{\overline{\mathbf{Y}}}$ can be considered as a valid representation of the tangent vector $\xi_{\overline{\mathbf{Y}}}$. To circumvent the aforementioned problem and obtain a unique representation of $\xi_{\overline{\mathbf{Y}}}$ for each predecessor $\mathbf{Y} \in \pi^{-1}(\overline{\mathbf{Y}})$, we use the fact that $\pi^{-1}(\overline{\mathbf{Y}})$ represents a manifold. Therefore, one can obtain a unique representation by orthogonally decomposing the tangent space $\mathcal{T}_{\mathbf{Y}} \mathcal{M}$ into a vertical space $\mathcal{V}_{\mathbf{Y}} \mathcal{M}$ and a horizontal space $\mathcal{H}_{\mathbf{Y}} \mathcal{M}$ such that

$$
\begin{aligned}
& \mathcal{V}_{\mathbf{Y}} \mathcal{M}=\mathcal{T}_{\mathbf{Y}} \pi^{-1}(\overline{\mathbf{Y}}) \\
& \mathcal{T}_{\mathbf{Y}} \mathcal{M}=\mathcal{V}_{\mathbf{Y}} \mathcal{M} \oplus \mathcal{H}_{\mathbf{Y}} \mathcal{M} .
\end{aligned}
$$

The ambient vector space can be composed into a tangent space $\mathcal{T}_{\mathbf{Y}} \mathcal{M}$ and its orthogonal complement $\mathcal{T}_{\mathbf{Y}} \mathcal{M}$. In particular, for each $\mathbf{Y} \in \mathcal{M}$, the embedding space $\mathbb{R}^{n \times p}$ can be uniquely decomposed into a direct sum of the above defined linear space, i.e.,

$$
\mathbb{R}^{n \times p}=\mathcal{H}_{\mathbf{Y}} \mathcal{M} \oplus \mathcal{V}_{\mathbf{Y}} \mathcal{M} \oplus \mathcal{T}_{\mathbf{Y}}^{\perp} \mathcal{M}
$$

The representation of $\xi_{\overline{\mathbf{Y}}} \in \mathcal{T}_{\overline{\mathbf{Y}}} \overline{\mathcal{M}}$ at $\mathbf{Y} \in \pi^{-1}(\overline{\mathbf{Y}})$, denoted by $\bar{\xi}_{\mathbf{Y}}$ and referred to as the horizontal lift of a tangent vector $\xi_{\overline{\mathbf{Y}}}$ at $\mathbf{Y}$, is the unique element in the horizontal space $\mathcal{H}_{\mathbf{Y}} \mathcal{M}$ satisfying $\mathrm{D}(\pi(\mathbf{Y}))\left[\bar{\xi}_{\mathbf{Y}}\right]=\xi_{\overline{\mathbf{Y}}}$. Such representation as horizontal lift allows to get a unique parameterization of tangent vectors in a quotient manifold. The manifold $\overline{\mathcal{M}}$ represents a Riemannian manifold for the Riemmanian metric $\langle., .\rangle_{\overline{\mathbf{Y}}}$ on $\mathcal{T}_{\overline{\mathbf{Y}}} \overline{\mathcal{M}}$ if and only if for all tangent vectors $\xi_{\overline{\mathbf{Y}}}, \eta_{\overline{\mathbf{Y}}} \in \mathcal{T}_{\overline{\mathbf{Y}}} \mathcal{M}$ the following holds

$$
\left\langle\bar{\xi}_{\mathbf{Y}_{1}}, \bar{\eta}_{\mathbf{Y}_{1}}\right\rangle_{\mathbf{Y}_{1}}=\left\langle\bar{\xi}_{\mathbf{Y}_{2}}, \bar{\eta}_{\mathbf{Y}_{2}}\right\rangle_{\mathbf{Y}_{2}}, \forall \mathbf{Y}_{1}, \mathbf{Y}_{2} \in \pi^{-1}(\overline{\mathbf{Y}}) .
$$

Under the above assumption, the operator $\langle., .\rangle_{\overline{\mathbf{Y}}}$ on $\mathcal{T}_{\overline{\mathbf{Y}}} \overline{\mathcal{M}}$ defined by $\left\langle\xi_{\overline{\mathbf{Y}}}, \eta_{\overline{\mathbf{Y}}}\right\rangle_{\overline{\mathbf{Y}}}=\left\langle\bar{\xi}_{\mathbf{Y}}, \bar{\eta}_{\mathbf{Y}}\right\rangle_{\mathbf{Y}}$ for any $\mathbf{Y} \in$ $\pi^{-1}(\overline{\mathbf{Y}})$ represents a well-defined Riemannian metric for the quotient manifold $\overline{\mathcal{M}}$. Let $\mathcal{P}_{\mathbf{Y}}^{\mathcal{H}}$ be the orthogonal projection from the ambient space $\mathbb{R}^{n \times p}$ to the horizontal space $\mathcal{H}_{\mathbf{Y}} \mathcal{M}$ and let $f: \mathcal{M} \rightarrow \mathbb{R}$ be a function that is constant on each equivalence class $[\mathbf{Y}]$ for all $\mathbf{Y} \in \mathcal{M}$. The above function, said to be compatible with the equivalence relationship, induces a function $\bar{f}: \overline{\mathcal{M}} \rightarrow \mathbb{R}$ such that $\bar{f}(\overline{\mathbf{Y}})=f(\mathbf{Y})$ for any predecessor $\mathbf{Y}$ of the equivalence class $\overline{\mathbf{Y}}$. Under the above assumptions, the Riemannian gradient is obtained by projecting the Euclidean one onto the horizontal space of any predecessor, 
i.e.,

$\operatorname{grad} \bar{f}(\overline{\mathbf{Y}})=\mathcal{P}_{\mathbf{Y}}^{\mathcal{H}}(\operatorname{Grad} f(\mathbf{Y})), \quad \mathbf{Y} \in \pi^{-1}(\overline{\mathbf{Y}})$.

In the same manner as for the embedded manifold, the Riemannian Hessian can be expressed as the covariant derivative of the Riemannian gradient on the quotient manifold hess $\bar{f}(\overline{\mathbf{Y}})\left[\xi_{\overline{\mathbf{Y}}}\right]=\nabla_{\bar{\xi}_{\mathbf{Y}}}$ grad $\bar{f}(\overline{\mathbf{Y}})$. Given that the ambient space, $\mathbb{R}^{n \times p}$ herein, is a vector space and that the Riemannian metric is induced and compatible, the connection simplifies as $\nabla_{\xi_{\overline{\mathbf{Y}}}} \eta_{\overline{\mathbf{Y}}}=\mathcal{P}_{\mathbf{Y}}^{\mathcal{H}}\left(\mathrm{D}\left(\eta_{\overline{\mathbf{Y}}}\right)\left[\bar{\xi}_{\mathbf{Y}}\right]\right)$, for any $\mathbf{Y} \in \pi^{-1}(\overline{\mathbf{Y}})$ which allows the Riemannian Hessian to be expressed as

$$
\text { hess } \bar{f}(\overline{\mathbf{Y}})\left[\xi_{\overline{\mathbf{Y}}}\right]=\mathcal{P}_{\mathbf{Y}}^{\mathcal{H}}\left(\mathrm{D}(\operatorname{grad} \bar{f}(\overline{\mathbf{Y}}))\left[\bar{\xi}_{\mathbf{Y}}\right]\right) \text {. }
$$

Let $\overline{\mathbf{Y}} \in \overline{\mathcal{M}}$ and $\mathbf{Y}_{1}$ and $\mathbf{Y}_{2}$ be any two arbitrary representatives in $\pi^{-1}(\overline{\mathbf{Y}})$. Assume that the retractions $\mathrm{R}_{\mathbf{Y}_{1}}$ and $\mathrm{R}_{\mathbf{Y}_{2}}$ on the tangent spaces $\mathcal{T}_{\mathbf{Y}_{1}} \mathcal{M}$ and $\mathcal{T}_{\mathbf{Y}_{2}} \mathcal{M}$ of the manifold $\mathcal{M}$ satisfy the property $\pi\left(\mathrm{R}_{\mathbf{Y}_{1}}\left(\bar{\xi}_{\mathbf{Y}_{1}}\right)\right)=$ $\pi\left(\mathbf{R}_{\mathbf{Y}_{2}}\left(\bar{\xi}_{\mathbf{Y}_{2}}\right)\right)$ for all tangent vectors. Such retraction is said to be compatible with the equivalence relationship and generate a retraction on the quotient manifold as follows

$$
\mathbf{R}_{\overline{\mathbf{Y}}}\left(\xi_{\overline{\mathbf{Y}}}\right)=\pi\left(\mathbf{R}_{\mathbf{Y}}\left(\bar{\xi}_{\mathbf{Y}}\right)\right), \mathbf{Y} \in \pi^{-1}(\overline{\mathbf{Y}})
$$

\section{Geometry of THE Embedded AND QuO- tient Low-Rank Positive Multinomial MANIFOLDS}

This section studies the geometries of the embedded and quotient low-rank positive multinomial manifolds. Subsection 4.1 derives the expression of the tangent space, Riemannian gradient, retraction, and Riemannian Hessian for the embedded low-rank positive multinomial manifold. Similarly, Subsection 4.2 derives these ingredients for the quotient low-rank positive multinomial manifold. The geometries of both manifolds are utilized in Section 5 to design first and second-order Riemannian algorithms for the problem of interest identified in Section 2.

\subsection{The Low-Rank Positive Multinomial Manifold}

The embedded low-rank positive multinomial manifold $\mathcal{M}_{p}^{n}$ is defined as $\mathcal{M}_{p}^{n}=\left\{\mathbf{Y} \in \mathbb{R}_{*}^{n \times p} \mid \mathbf{Y Y}^{\mathrm{T}}>\right.$ $\mathbf{0}$ and $\left.\mathbf{Y Y} \mathbf{Y}^{\mathrm{T}} \mathbf{1}=\mathbf{1}\right\}$. The manifold $\mathcal{M}_{p}^{n}$ is seen as an embedded manifold of the set of non-singular matrices $\mathbb{R}_{*}^{n \times p}$. In other words, the manifold is regarded as an embedded structure in the non-compact Stiefel manifold $\mathbb{R}_{*}^{n \times p}$. Define the function $f(\mathbf{Y})=g\left(\mathbf{Y} \mathbf{Y}^{\mathrm{T}}\right)$ wherein the function $g$ is defined in (2).

The tangent space $\mathcal{T}_{\mathbf{Y}} \mathcal{M}_{p}^{n}$ for a point $\mathbf{Y} \in \mathcal{M}_{p}^{n}$ is computed using a two-way inclusion. For an open interval $I$ containing 0 , let $\gamma: I \subseteq \mathbb{R} \rightarrow \mathcal{M}_{p}^{n}$ be a smooth curve in the embedded low-rank positive multinomial manifold satisfying $\gamma(0)=\mathbf{Y}$ and $\dot{\gamma}(0)=\left.\frac{d \gamma(t)}{d t}\right|_{t=0}=\xi_{\mathbf{Y}}$. The curve $\gamma(t)$ being in the manifold for $t \in I$, it satisfies $\gamma(t) \gamma(t)^{\mathrm{T}} \mathbf{1}=\mathbf{1}$. Taking derivative of the equation above, evaluating at $t=0$, and substituting the values for $\gamma(0)$ and $\dot{\gamma}(0)$ yields the following characterization for the tangent space at $\mathbf{Y} \in \mathcal{M}_{p}^{n}$

$$
\mathcal{T}_{\mathbf{Y}} \mathcal{M}_{p}^{n} \subseteq\left\{\xi_{\mathbf{Y}} \in \mathbb{R}^{n \times p} \mid\left(\xi_{\mathbf{Y}} \mathbf{Y}^{\mathrm{T}}+\mathbf{Y} \xi_{\mathbf{Y}}^{\mathrm{T}}\right) \mathbf{1}=\mathbf{0}\right\} .
$$

The converse is obtained by extending the tangent space characterization of [17]. To that purpose, first introduce the rank and level set of a function. Let $F: \mathcal{M}_{1} \rightarrow \mathcal{M}_{2}$ be a smooth function from a manifold $\mathcal{M}_{1}$ of dimension $d_{1}$ to the manifold $\mathcal{M}_{2}$ of dimension $d_{2}$. The rank of $F$ at a point $\mathbf{X} \in \mathcal{M}_{1}$ is the dimension of the image of $\mathcal{T}_{\mathbf{X}} \mathcal{M}_{1}$ by the indefinite directional derivative $\mathrm{D}(F(\mathbf{X}))[]:. \mathcal{T}_{\mathbf{X}} \mathcal{M}_{1} \rightarrow \mathcal{M}_{2}$, i.e., the rank is defined as the dimension of the manifold generated by $\mathrm{D}(F(\mathbf{X}))\left[\xi_{\mathbf{X}}\right]$ for all $\xi_{\mathbf{X}} \in \mathcal{T}_{\mathbf{X}} \mathcal{M}_{1}$. A function $F$ is rank-constant if the rank is constant for all $\mathbf{X} \in \mathcal{M}_{1}$. Given $\mathbf{Y} \in \mathcal{M}_{2}$, the set $F^{-1}(\mathbf{Y})$ is called a level set or a fiber of $F$.

While the authors in [17] present a precise characterization of the tangent space of manifolds that can be expressed as a level set of some function, the following lemma extends the results for manifolds that contain the level set of some function.

Lemma 1. Let $\mathcal{M}$ be a manifold embedded in the Euclidean space $\mathcal{E}$ and let $\mathcal{E}^{*}$ be an open subset of $\mathcal{E}$. Assuming that $\mathcal{M}$ contains the level set of a rank-constant function $F: \mathcal{E}^{*} \rightarrow \mathbb{R}^{n}$, the tangent space $\mathcal{T}_{\mathbf{X}} \mathcal{M}$ at $\mathbf{X} \in \mathcal{M}$ satisfies $\operatorname{Ker}(D(F(\mathbf{X}))) \subseteq \mathcal{T}_{\mathbf{X}} \mathcal{M}$.

Proof: The manifold $\mathcal{M}$ contains the level set of $F$. Let $\mathbf{Y}$ be the fiber such that $F^{-1}(\mathbf{Y}) \subseteq \mathcal{M}$ and define $\mathcal{M}^{\prime}$ as $F^{-1}(\mathbf{Y})=\mathcal{M}^{\prime}$. Since $\mathcal{M}^{\prime}$ is a level-set of a smooth function, it represents a manifold. Note that $\mathcal{M}^{\prime}$ satisfies the conditions of Proposition 3.3.3 of [17] as it is a level set of a constant-rank function. Therefore, we obtain $\operatorname{Ker}(\mathrm{D}(F(\mathbf{X})))=\mathcal{T}_{\mathbf{X}} \mathcal{M}^{\prime}$. Finally, by definition of $\mathcal{M}^{\prime}$, we have $\mathcal{M}^{\prime} \subseteq \mathcal{M}$ which implies that $\mathcal{T}_{\mathbf{X}} \mathcal{M}^{\prime} \subseteq \mathcal{T}_{\mathbf{X}} \mathcal{M}$. Exploiting this last inequality yields the result $\operatorname{Ker}(\mathrm{D}(F(\mathbf{X}))) \subseteq \mathcal{T}_{\mathbf{X}} \mathcal{M}$.

Let $\mathbb{R}_{*+}^{n \times p}$ be the set of entry-wise positive and full rank $n \times p$ matrices and define $F: \mathbb{R}_{*+}^{n \times p} \rightarrow \mathbb{R}^{n}$ such that $F(\mathbf{Y})=\mathbf{Y Y}^{\mathrm{T}} \mathbf{1}$. Given that $\mathbf{Y} \in \mathbb{R}_{*+}^{n \times p}$ with positive entries, it is clear that $\mathbf{Y} \mathbf{Y}^{\mathrm{T}}>\mathbf{0}$. Therefore, the manifold $\mathcal{M}_{p}^{n}$ includes the predecessor of $\mathbf{1}$ by $F$, i.e., $F^{-1}(\mathbf{1}) \subseteq$ $\mathcal{M}_{p}^{n}$. The fact that $F$ has a fixed rank can be readily obtained from the fact that 1 is a regular value of $F$, i.e., the rank of any $\mathbf{Y} \in F^{-1}(\mathbf{1})$ is equal to $n$. Using the result of Lemma 1, we conclude that

$$
\begin{aligned}
\operatorname{Ker}(\mathrm{D}(F(\mathbf{Y}))) & \subseteq \mathcal{T}_{\mathbf{Y}} \mathcal{M}_{p}^{n} \Leftrightarrow \\
\left\{\xi_{\mathbf{Y}} \in \mathbb{R}^{n \times p} \mid \mathrm{D}(F(\mathbf{Y}))\left[\xi_{\mathbf{Y}}\right]=\mathbf{0}\right\} & \subseteq \mathcal{T}_{\mathbf{Y}} \mathcal{M}_{p}^{n} \Leftrightarrow \\
\left\{\xi_{\mathbf{Y}} \in \mathbb{R}^{n \times p} \mid\left(\xi_{\mathbf{Y}} \mathbf{Y}^{\mathrm{T}}+\mathbf{Y} \xi_{\mathbf{Y}}^{\mathrm{T}}\right) \mathbf{1}=\mathbf{0}\right\} & \subseteq \mathcal{T}_{\mathbf{Y}} \mathcal{M}_{p}^{n} .
\end{aligned}
$$

Combining (12) and (13) allows to conclude that the tangent space $\mathcal{T}_{\mathbf{Y}} \mathcal{M}_{p}^{n}$ for a point $\mathbf{Y} \in \mathcal{M}_{p}^{n}$ is given by

$$
\mathcal{T}_{\mathbf{Y}} \mathcal{M}_{p}^{n}=\left\{\xi_{\mathbf{Y}} \in \mathbb{R}^{n \times p} \mid\left(\xi_{\mathbf{Y}} \mathbf{Y}^{\mathrm{T}}+\mathbf{Y} \xi_{\mathbf{Y}}^{\mathrm{T}}\right) \mathbf{1}=\mathbf{0}\right\} .
$$

The dimension of the tangent space, and thus the dimension of the manifold, can readily be seen from its characterization. The tangent space is embedded in a $n p$ dimension Euclidean space with $n$ independent linear equations which gives a dimension of $n(p-1)$. As 
stated in Section 3, this manuscript considers the induced Frobenius inner product, i.e., for $\xi_{\mathbf{Y}}, \eta_{\mathbf{Y}} \in \mathcal{T}_{\mathbf{Y}} \mathcal{M}_{p}^{n}$ we have $\left\langle\xi_{\mathbf{Y}}, \eta_{\mathbf{Y}}\right\rangle_{\mathbf{Y}}=\operatorname{Tr}\left(\xi_{\mathbf{Y}}^{\mathrm{T}} \eta_{\mathbf{Y}}\right)$. The expression of the Riemannian gradient as a function of the Euclidean gradient is given in the following proposition

Proposition 2. Let Grad $f(\mathbf{Y})$ be the Euclidean gradient of $f$ at $\mathbf{Y}$, the Riemannian gradient grad $f(\mathbf{Y})$ is given by

$$
\text { grad } f(\mathbf{Y})=\text { Grad } f(\mathbf{Y})-\left(\alpha \mathbf{1}^{\mathrm{T}}+\mathbf{1} \alpha^{\mathrm{T}}\right) \mathbf{Y},
$$

with $\alpha$ being the $n$-dimensional vector obtained by

$$
\begin{aligned}
\alpha=\frac{1}{n}\left(\mathbf{I}-\frac{1}{2 n} \mathbf{1 1}^{\mathrm{T}}\right)\left(\mathbf{I}+\mathbf{Y} \mathbf{Y}^{\mathrm{T}}\right)^{-1} \times \\
\left(\operatorname{Grad} f(\mathbf{Y}) \mathbf{Y}^{\mathrm{T}}+\mathbf{Y G r a d} f(\mathbf{Y})^{\mathrm{T}}\right) \mathbf{1} .
\end{aligned}
$$

Proof: Define $\mathcal{P}_{\mathbf{Y}}$ as the orthogonal projection from the ambient space $\mathbb{R}^{n \times p}$ to the tangent space $\mathcal{T}_{\mathbf{Y}} \mathcal{M}_{p}^{n}$. As stated earlier, the Riemannian gradient at $\mathbf{Y}$ can be expressed as the orthogonal projection of the Euclidean gradient at $\mathbf{Y}$ onto the tangent space $\mathcal{T}_{\mathbf{Y}} \mathcal{M}_{p}^{n}$ [17]. In order to derive an expression of the orthogonal projection $\mathcal{P}_{\mathbf{Y}}$, this proof first parametrizes the orthogonal complement of the tangent space. The orthogonal complement $\mathcal{T}_{\mathbf{Y}}^{\perp} \mathcal{M}_{p}^{n}$ of the tangent space $\mathcal{T}_{\mathbf{Y}} \mathcal{M}_{p}^{n}$ in $\mathbb{R}^{n \times p}$ is parametrized by

$$
\mathcal{T}_{\mathbf{Y}}^{\perp} \mathcal{M}_{p}^{n}=\left\{\eta \in \mathbb{R}^{n \times p} \mid \eta=\left(\alpha \mathbf{1}^{\mathrm{T}}+\mathbf{1} \alpha^{\mathrm{T}}\right) \mathbf{Y}, \alpha \in \mathbb{R}^{n}\right\},
$$

Indeed, let $\xi_{\mathbf{Y}} \in \mathcal{T}_{\mathbf{Y}} \mathcal{M}_{p}^{n}$ and $\eta_{\mathbf{Y}}=\left(\alpha \mathbf{1}^{\mathrm{T}}+\mathbf{1} \alpha^{\mathrm{T}}\right) \mathbf{Y}$, we obtain

$$
\begin{aligned}
\left\langle\xi_{\mathbf{Y}}, \eta_{\mathbf{Y}}\right\rangle_{\mathbf{Y}} & =\operatorname{Tr}\left(\xi_{\mathbf{Y}}^{\mathrm{T}}\left(\alpha \mathbf{1}^{\mathrm{T}}+\mathbf{1} \alpha^{\mathrm{T}}\right) \mathbf{Y}\right) \\
& =\operatorname{Tr}\left(\xi_{\mathbf{Y}} \mathbf{Y}^{\mathrm{T}} \mathbf{1} \alpha^{\mathrm{T}}\right)+\operatorname{Tr}\left(\mathbf{Y} \xi_{\mathbf{Y}}^{\mathrm{T}} \mathbf{1} \alpha^{\mathrm{T}}\right) \\
& \stackrel{(a)}{=} \operatorname{Tr}\left(\xi_{\mathbf{Y}} \mathbf{Y}^{\mathrm{T}} \mathbf{1} \alpha^{\mathrm{T}}\right)-\operatorname{Tr}\left(\xi_{\mathbf{Y}} \mathbf{Y}^{\mathrm{T}} \mathbf{1} \alpha^{\mathrm{T}}\right)=0,
\end{aligned}
$$

wherein the equality in $(a)$ is obtained from the fact that $\xi_{\mathbf{Y}}$ satisfies $\left(\xi_{\mathbf{Y}} \mathbf{Y}^{\mathrm{T}}+\mathbf{Y} \xi_{\mathbf{Y}}^{\mathrm{T}}\right) \mathbf{1}=\mathbf{0}$. Therefore, we obtain that $\left\{\eta \in \mathbb{R}^{n \times p} \mid \eta=\left(\alpha \mathbf{1}^{\mathrm{T}}+\mathbf{1} \alpha^{\mathrm{T}}\right) \mathbf{Y}, \alpha \in\right.$ $\left.\mathbb{R}^{n}\right\}, \subseteq \mathcal{T}_{\mathbf{Y}}^{\perp} \mathcal{M}_{p}^{n}$. Finally, note that $\operatorname{Dim}\left(\left\{\eta \in \mathbb{R}^{n \times p} \mid \eta=\right.\right.$ $\left.\left.\left(\alpha \mathbf{1}^{\mathrm{T}}+\mathbf{1} \alpha^{\mathrm{T}}\right) \mathbf{Y}, \alpha \in \mathbb{R}^{n}\right\}\right)=n$ which in addition to the dimension of tangent space of $n(p-1)$ concludes that $\mathcal{T}_{\mathbf{Y}}^{\perp} \mathcal{M}_{p}^{n}=\left\{\eta \in \mathbb{R}^{n \times p} \mid \eta=\left(\alpha \mathbf{1}^{\mathrm{T}}+\mathbf{1} \alpha^{\mathrm{T}}\right) \mathbf{Y}, \alpha \in \mathbb{R}^{n}\right\}$.

We now derive an explicit expression for the orthogonal projection $\mathcal{P}_{\mathbf{Y}}$ onto the tangent space $\mathcal{T}_{\mathbf{Y}} \mathcal{M}_{p}^{n}$. Let $\mathbf{Z} \in \mathbb{R}^{n \times p}$ be a point on the ambient space written as the sum of the component in the tangent space $\mathcal{T}_{\mathbf{Y}} \mathcal{M}_{p}^{n}$ and its orthogonal complement as $\mathbf{Z}=\mathcal{P}_{\mathbf{Y}}(\mathbf{Z})+\mathcal{P}_{\mathbf{Y}}(\mathbf{Z})$. Recall that a point $\mathcal{P}_{\mathbf{Y}}(\mathbf{Z})$ in the tangent space $\mathcal{T}_{\mathbf{Y}} \mathcal{M}_{p}^{n}$ satisfies $\left(\mathcal{P}_{\mathbf{Y}}(\mathbf{Z}) \mathbf{Y}^{\mathrm{T}}+\mathbf{Y} \mathcal{P}_{\mathbf{Y}}(\mathbf{Z})^{\mathrm{T}}\right) \mathbf{1}=\mathbf{0}$. Furthermore, a point $\mathcal{P}_{\mathbf{Y}}(\mathbf{Z})$ on the orthogonal complement of the tangent space is parametrized by $\mathcal{P}_{\mathbf{Y}}^{\perp}(\mathbf{Z})=\left(\alpha \mathbf{1}^{\mathrm{T}}+\mathbf{1} \alpha^{\mathrm{T}}\right) \mathbf{Y}$. Therefore, we obtain

$$
\begin{aligned}
\left(\mathbf{Z} \mathbf{Y}^{\mathrm{T}}\right. & \left.+\mathbf{Y} \mathbf{Z}^{\mathrm{T}}\right) \mathbf{1}=\left(\mathcal{P}_{\mathbf{Y}}^{\perp}(\mathbf{Z}) \mathbf{Y}^{\mathrm{T}}+\mathbf{Y} \mathcal{P}_{\mathbf{Y}}^{\perp}(\mathbf{Z})^{\mathrm{T}}\right) \mathbf{1} \\
& =\left(\left(\alpha \mathbf{1}^{\mathrm{T}}+\mathbf{1} \alpha^{\mathrm{T}}\right) \mathbf{Y} \mathbf{Y}^{\mathrm{T}}+\mathbf{Y} \mathbf{Y}^{\mathrm{T}}\left(\alpha \mathbf{1}^{\mathrm{T}}+\mathbf{1} \alpha^{\mathrm{T}}\right)\right) \mathbf{1} \\
& \stackrel{(\mathrm{a})}{=} n \alpha+\mathbf{1 1}^{\mathrm{T}} \alpha+\mathbf{Y} \mathbf{Y}^{\mathrm{T}}\left(n \alpha+\mathbf{1} \mathbf{1}^{\mathrm{T}} \alpha\right) \\
& \stackrel{(\mathrm{b})}{=}\left(n \mathbf{I}+2 \mathbf{1 1} \mathbf{1}^{\mathrm{T}}+n \mathbf{Y} \mathbf{Y}^{\mathrm{T}}\right) \alpha,
\end{aligned}
$$

with both equalities in $(a)$ and $(b)$ being obtained from the fact that $\mathbf{Y Y}^{\mathrm{T}} \mathbf{1}=\mathbf{1}$. Finally, notice the following identities

$$
\begin{aligned}
\left(\mathbf{I}+\mathbf{Y} \mathbf{Y}^{\mathrm{T}}\right)\left(n \mathbf{I}+\mathbf{1 1}^{\mathrm{T}}\right) & =n \mathbf{I}+2 \mathbf{1 1}{ }^{\mathrm{T}}+n \mathbf{Y} \mathbf{Y}^{\mathrm{T}} \\
\left(n \mathbf{I}+\mathbf{1 1}^{\mathrm{T}}\right)^{-1} & =\frac{1}{n}\left(\mathbf{I}-\frac{1}{2 n} \mathbf{1 1}^{\mathrm{T}}\right) .
\end{aligned}
$$

Substituting (17a) and (17b) in (16) gives an expression for $\alpha$ as

$$
\alpha=\frac{1}{n}\left(\mathbf{I}-\frac{1}{2 n} \mathbf{1 1}^{\mathrm{T}}\right)\left(\mathbf{I}+\mathbf{Y} \mathbf{Y}^{\mathrm{T}}\right)^{-1}\left(\mathbf{Z} \mathbf{Y}^{\mathrm{T}}+\mathbf{Y} \mathbf{Z}^{\mathrm{T}}\right) \mathbf{1} .
$$

Therefore, the orthogonal projection $\mathcal{P}_{\mathbf{Y}}$ from the ambient space $\mathbb{R}^{n \times p}$ onto the tangent space $\mathcal{T}_{\mathbf{Y}} \mathcal{M}_{p}^{n}$ is given by

with $\alpha$ defined by

$$
\mathcal{P}_{\mathbf{Y}}(\mathbf{Z})=\mathbf{Z}-\left(\alpha \mathbf{1}^{\mathrm{T}}+\mathbf{1} \alpha^{\mathrm{T}}\right) \mathbf{Y}
$$

$$
\alpha=\frac{1}{n}\left(\mathbf{I}-\frac{1}{2 n} \mathbf{1 1}^{\mathrm{T}}\right)\left(\mathbf{I}+\mathbf{Y} \mathbf{Y}^{\mathrm{T}}\right)^{-1}\left(\mathbf{Z} \mathbf{Y}^{\mathrm{T}}+\mathbf{Y} \mathbf{Z}^{\mathrm{T}}\right) \mathbf{1} .
$$

As stated earlier, the final expression of the Riemannian gradient is obtained by projecting the Euclidean one onto the tangent space, i.e., grad $f(\mathbf{Y})=$ $\mathcal{P}_{\mathbf{Y}}($ Grad $f(\mathbf{Y}))$. Using the expression of the orthogonal projection, the Riemannian gradient can be expressed as grad $f(\mathbf{Y})=$ Grad $f(\mathbf{Y})-\left(\alpha \mathbf{1}^{\mathrm{T}}+\mathbf{1} \alpha^{\mathrm{T}}\right) \mathbf{Y}$,

with $\alpha$ being the $n$-dimensional vector obtained by

$$
\begin{aligned}
& \alpha=\frac{1}{n}(\mathbf{I}-\left.\frac{1}{2 n} \mathbf{1 1}^{\mathrm{T}}\right)\left(\mathbf{I}+\mathbf{Y} \mathbf{Y}^{\mathrm{T}}\right)^{-1}\left(\operatorname{Grad} f(\mathbf{Y}) \mathbf{Y}^{\mathrm{T}}\right. \\
&\left.+\mathbf{Y G r a d} f(\mathbf{Y})^{\mathrm{T}}\right) \mathbf{1} .
\end{aligned}
$$

As stated in Section 3, the Riemannian Hessian $\operatorname{hess} f(\mathbf{Y})\left[\xi_{\mathbf{Y}}\right]$ can be obtained by projecting the directional derivation $\mathrm{D}(\operatorname{grad} f(\mathbf{Y}))\left[\xi_{\mathbf{Y}}\right]$ onto the tangent space $\mathcal{T}_{\mathbf{Y}} \mathcal{M}_{p}^{n}$, i.e., $\operatorname{hess} f(\mathbf{Y})\left[\xi_{\mathbf{Y}}\right]=$ $\mathcal{P}_{\mathbf{Y}}\left(\mathrm{D}(\operatorname{grad} f(\mathbf{Y}))\left[\xi_{\mathbf{Y}}\right]\right)$. Using the fact that $\mathrm{D}(\operatorname{Grad} f(\mathbf{Y}))\left[\xi_{\mathbf{Y}}\right]=\operatorname{Hess} f(\mathbf{Y})\left[\xi_{\mathbf{Y}}\right]$, the directional derivative of the Riemannian gradient is

$$
\begin{array}{r}
\mathrm{D}(\operatorname{grad} f(\mathbf{Y}))\left[\xi_{\mathbf{Y}}\right]=\operatorname{Hess} f(\mathbf{Y})\left[\xi_{\mathbf{Y}}\right]-\left(\dot{\alpha} \mathbf{1}^{\mathrm{T}}+\mathbf{1} \dot{\alpha}^{\mathrm{T}}\right) \mathbf{Y} \\
-\left(\alpha \mathbf{1}^{\mathrm{T}}+\mathbf{1} \alpha^{\mathrm{T}}\right) \xi_{\mathbf{Y}},
\end{array}
$$

with $\dot{\alpha}$ being defined by $\dot{\alpha}=\mathrm{D}(\alpha)\left[\xi_{\mathbf{Y}}\right]$. Define the constant matrix $\mathbf{A}=\frac{1}{n}\left(\mathbf{I}-\frac{1}{2 n} \mathbf{1 1}^{\mathrm{T}}\right)$ and recall that $\alpha$ is defined by

$\alpha=\mathbf{A}\left(\mathbf{I}+\mathbf{Y} \mathbf{Y}^{\mathrm{T}}\right)^{-1}\left(\operatorname{Grad} f(\mathbf{Y}) \mathbf{Y}^{\mathrm{T}}+\mathbf{Y G r a d} f(\mathbf{Y})^{\mathrm{T}}\right) \mathbf{1}$.

The directional derivative of the quantity $\left(\mathbf{I}+\mathbf{Y Y}^{\mathrm{T}}\right)^{-1}=$ B can be obtained by exploiting the Kailath variant of Sherman-Morrison-Woodbury formula [36] which gives

$$
\dot{\mathbf{B}}=\mathrm{D}(\mathbf{B})\left[\xi_{\mathbf{Y}}\right]=-\mathbf{B}\left(\mathbf{Y} \xi_{\mathbf{Y}}^{T}+\xi_{\mathbf{Y}} \mathbf{Y}^{T}\right) \mathbf{B} \text {. }
$$

Let the vector $\beta=\left(\operatorname{Grad} f(\mathbf{Y}) \mathbf{Y}^{\mathrm{T}}+\mathbf{Y G r a d} f(\mathbf{Y})^{\mathrm{T}}\right) \mathbf{1}$. Its directional derivative is given by

$$
\begin{aligned}
\dot{\beta}= & \mathrm{D}(\beta)\left[\xi_{\mathbf{Y}}\right]=\left(\operatorname{Grad} f(\mathbf{Y}) \xi_{\mathbf{Y}}^{\mathrm{T}}+\xi_{\mathbf{Y}} \operatorname{Grad} f(\mathbf{Y})^{\mathrm{T}}\right. \\
& \left.+\operatorname{Hess} f(\mathbf{Y})\left[\xi_{\mathbf{Y}}\right] \mathbf{Y}^{\mathrm{T}}+\mathbf{Y H e s s} f(\mathbf{Y})\left[\xi_{\mathbf{Y}}\right]^{\mathrm{T}}\right) \mathbf{1} .
\end{aligned}
$$

Finally, the expression of $\dot{\alpha}$ simplifies to $\dot{\alpha}=\mathbf{A B}(\dot{\beta}-$ $\left.\left(\mathbf{Y} \xi_{\mathbf{Y}}^{T}+\xi_{\mathbf{Y}} \mathbf{Y}^{T}\right) \mathbf{B} \beta\right)$ which allows to get the Riemannian Hessian by applying the projection operator $\mathcal{P}_{\mathbf{Y}}$ to the 
expression in (18).

Let $R_{Y}$ denote a retraction from the tangent space $\mathcal{T}_{\mathbf{Y}} \mathcal{M}_{p}^{n}$ to the manifold $\mathcal{M}_{p}^{n}$. To derive an expression of such operator, recall that for an entry-wise positive matrix $\mathbf{A} \in \mathcal{S}^{n}$, there exists a unique diagonal matrix $\mathbf{D}$ with strictly positive entries such that $\mathbf{S}=$ DAD is a doubly stochastic matrix. Such matrix is obtained by the DAD algorithm [37]. This result extends the Sinkhorn's theorem for symmetric matrices [38].

Let $\mathbb{R}_{+/ 2}^{n \times p}=\left\{\mathbf{Z} \in \mathbb{R}^{n \times p} \mid \mathbf{Z Z}^{\mathrm{T}}>0\right\}$ and introduce the projection $\Pi: \mathbb{R}_{+/ 2}^{n \times p} \rightarrow \mathcal{M}_{p}^{n}$ defined by $\Pi(\mathbf{Z})=$ $\mathbf{D Z}$ wherein the diagonal matrix $\mathbf{D}$ is obtained from applying the DAD algorithm to the matrix $\mathbf{Z Z}^{\mathrm{T}}$. This paper suggests the following retraction to project tangent vectors to the manifold

Theorem 3. Let $R_{\mathbf{Y}}: \mathcal{T}_{\mathbf{Y}} \mathcal{M}_{p}^{n} \rightarrow \mathcal{M}_{p}^{n}$ be defined by

$$
R_{\mathbf{Y}}\left(\xi_{\mathbf{Y}}\right)=\Pi\left(\mathbf{Y}+\mathbf{1}_{n} \mathbf{1}_{p}^{\mathrm{T}}-\exp \left(-\xi_{\mathbf{Y}}\right)\right)
$$

with $\exp \left(\xi_{\mathbf{Y}}\right)$ begin the entry-wise exponential of the entries of the matrix $\xi_{\mathbf{Y}}$. The operator $R_{\mathbf{Y}}$ is a well-defined retraction from the neighborhood $\mathcal{N}_{\mathbf{0}}$ of $\mathcal{T}_{\mathbf{Y}} \mathcal{M}_{p}^{n}$ to $\mathcal{M}_{p}^{n}$.

Proof: In order to show that the operator suggested in the theorem represents a well-defined retraction, one needs to show that there exists an open interval $\mathcal{N}_{\mathbf{0}}$ around $\mathbf{0}$ such that for all tangent vectors $\xi_{\mathbf{Y}}$ in $\mathcal{N}_{\mathbf{0}} \subset$ $\mathcal{T}_{\mathbf{Y}} \mathcal{M}_{p}^{n}$, for some neighborhood $\mathcal{N}_{\mathbf{0}}$, the following properties are satisfied

$$
\begin{aligned}
\mathbf{R}_{\mathbf{Y}}\left(\xi_{\mathbf{Y}}\right) & \in \mathcal{M}_{p}^{n} \\
\mathbf{R}_{\mathbf{Y}}(\mathbf{0}) & =\mathbf{Y} \\
\left.\frac{d \mathbf{R}_{\mathbf{Y}}\left(\tau \xi_{\mathbf{Y}}\right)}{d \tau}\right|_{\tau=0} & =\xi_{\mathbf{Y}} .
\end{aligned}
$$

By definition of the retraction and the projection operator $\Pi$, it is sufficient to show that there exists a neighborhood $\mathcal{N}_{\mathbf{0}}$ such that the matrix $\mathbf{Y}+\mathbf{1}_{n} \mathbf{1}_{p}^{\mathrm{T}}-\exp \left(-\xi_{\mathbf{Y}}\right)$ is in the set $\mathbb{R}_{+/ 2}^{n \times p}$ to conclude that $\mathbf{R}_{\mathbf{Y}}\left(\xi_{\mathbf{Y}}\right)$ satisfies (20a). Let $x_{n}$ be a real sequence converging to zero and define the matrix sequence $y_{n}=\mathbf{Y}+\mathbf{1}_{n} \mathbf{1}_{p}^{\mathrm{T}}-\exp \left(-x_{n} \xi_{\mathbf{Y}}\right)$. Therefore, the matrix sequence $y_{n}$ converges to $\mathbf{Y} \in \mathbb{R}_{+/ 2}^{n \times p}$. The set $\mathbb{R}_{+/ 2}^{n \times p}$ being both open and Hausdorff, there exists $N \in \mathbb{N}$ such that for all $n \geq N$, we have $y_{n} \in \mathbb{R}_{+/ 2}^{n \times p}$ which allows to conclude that, for small enough $\xi_{\mathbf{Y}}$, we have $\mathrm{R}_{\mathbf{Y}}\left(\xi_{\mathbf{Y}}\right) \in \mathcal{M}_{p}^{n}$.

The centering property (20b) can readily be seen from the definition of the retraction. Indeed, given that $e^{0}=1$, it is clear that $R_{\mathbf{Y}}(\mathbf{0})=\mathbf{Y}$. Finally, the local rigidity property (20c) is obtained from the study of the first order perturbation of the projection operator $\Pi$. Note that the curve $\gamma(t)=\mathbf{Y}+\mathbf{1}_{n} \mathbf{1}_{p}^{\mathrm{T}}-\exp \left(-t \xi_{\mathbf{Y}}\right)$ has a first order approximation of $\gamma(t)=\mathbf{Y}+t \xi_{\mathbf{Y}}+o\left(t^{2}\right)$. Therefore, to obtain the derivative of $\mathbf{R}_{\mathbf{Y}}\left(\tau \xi_{\mathbf{Y}}\right)$ around 0 , it is sufficient to study the behavior of $\Pi$ for small perturbations in the tangent space $\mathcal{T}_{\mathbf{Y}} \mathcal{M}_{p}^{n}$. Recall that $\Pi(\mathbf{Z})=\mathbf{D Z}$. Therefore, the first order perturbation is given by the diagonal matrix $\delta \mathbf{D}$ such that $\Pi(\mathbf{Z}+\delta \mathbf{Z})=(\mathbf{D}+\delta \mathbf{D})(\mathbf{Z}+\delta \mathbf{Z})$. Since $\mathbf{Y} \in \mathcal{M}_{p}^{n}$, then $\Pi(\mathbf{Y})=\mathbf{Y}$, i.e., $\mathbf{D}=\mathbf{I}$. Note that the first order perturbation of $\Pi\left(\mathbf{Y}+\xi_{\mathbf{Y}}\right)$ can be obtained through the study of the first order perturbation of the operator $\Pi\left(\mathbf{Y}+\xi_{\mathbf{Y}}\right) \Pi\left(\mathbf{Y}+\xi_{\mathbf{Y}}\right)^{\mathrm{T}}$. Indeed, let $F: \mathcal{M}_{1} \rightarrow \mathcal{M}_{2}$ be a differential function with a first order approximation $F(\mathbf{X}+$ $\delta \mathbf{X})=F(\mathbf{X})+\mathrm{D}(F(\mathbf{X}))[\delta \mathbf{X}]+o(\delta \mathbf{X})^{2}$. Then, the operator $F(.) F^{\mathrm{T}}($.$) has the following first order expansion F(\mathbf{X}+$ $\delta \mathbf{X}) F(\mathbf{X}+\delta \mathbf{X})^{\mathrm{T}}=F(\mathbf{X}) F(\mathbf{X})^{\mathrm{T}}+F(\mathbf{X}) \mathrm{D}(F(\mathbf{X}))[\delta \mathbf{X}]^{\mathrm{T}}+$ $\mathrm{D}(F(\mathbf{X}))[\delta \mathbf{X}] F(\mathbf{X})^{\mathrm{T}}+o(\delta \mathbf{X})^{2}$ which allows to readily get the coefficients of $F$. The first order perturbation of $\Pi\left(\mathbf{Y}+\xi_{\mathbf{Y}}\right) \Pi\left(\mathbf{Y}+\xi_{\mathbf{Y}}\right)^{\mathrm{T}}$ is given by

$$
\begin{aligned}
& \Pi\left(\mathbf{Y}+\xi_{\mathbf{Y}}\right) \Pi\left(\mathbf{Y}+\xi_{\mathbf{Y}}\right)^{\mathrm{T}} \\
& \quad=(\mathbf{I}+\delta \mathbf{D})\left(\mathbf{Y}+\xi_{\mathbf{Y}}\right)\left(\mathbf{Y}+\xi_{\mathbf{Y}}\right)^{\mathrm{T}}(\mathbf{I}+\delta \mathbf{D}) \\
& \quad \stackrel{(\mathrm{a})}{=} \mathbf{Y} \mathbf{Y}^{\mathrm{T}}+\mathbf{Y} \xi_{\mathbf{Y}}^{\mathrm{T}}+\mathbf{Y} \mathbf{Y}^{\mathrm{T}} \delta \mathbf{D}+\xi_{\mathbf{Y}} \mathbf{Y}^{\mathrm{T}}+\delta \mathbf{D Y} \mathbf{Y}^{\mathrm{T}},
\end{aligned}
$$

wherein the equality in $(a)$ only keeps the first order terms of the expansion. The following part of the proof uses the definition of the embedded manifold and its tangent space to conclude that $\delta \mathbf{D}=\mathbf{0}$. Recall that $\Pi(\mathbf{Z}) \Pi(\mathbf{Z})^{\mathrm{T}} \mathbf{1}=\mathbf{1}$ and $\left(\mathbf{Y} \xi_{\mathbf{Y}}^{\mathrm{T}}+\xi_{\mathbf{Y}} \mathbf{Y}^{\mathrm{T}}\right) \mathbf{1}=\mathbf{0}$ and define $\delta \mathbf{D} 1=\delta \mathbf{d}$. Multiplying (21) by 1 yields the following equation

$$
\mathbf{1}=\mathbf{1}+\mathbf{0}+\left(\mathbf{Y} \mathbf{Y}^{\mathrm{T}}+\mathbf{I}\right) \delta \mathbf{d} .
$$

Therefore, $\delta \mathbf{d}$ is in the null space of $\left(\mathbf{Y Y}^{\mathrm{T}}+\mathbf{I}\right)$. However, since $\mathbf{Y} \mathbf{Y}^{\mathrm{T}}$ is doubly stochastic, it is concluded that $\delta \mathbf{D}=\mathbf{0}$. Therefore, we obtain

$$
\Pi\left(\mathbf{Y}+\xi_{\mathbf{Y}}\right) \Pi\left(\mathbf{Y}+\xi_{\mathbf{Y}}\right)^{\mathrm{T}}=\mathbf{Y} \mathbf{Y}^{\mathrm{T}}+\mathbf{Y} \xi_{\mathbf{Y}}^{\mathrm{T}}+\xi_{\mathbf{Y}} \mathbf{Y}^{\mathrm{T}},
$$

which concludes that the first order perturbation of $\Pi$ is given by $\Pi\left(\mathbf{Y}+\xi_{\mathbf{Y}}\right)=\mathbf{Y}+\xi_{\mathbf{Y}}$. Therefore, we have $\mathbf{R}_{\mathbf{Y}}\left(\tau \xi_{\mathbf{Y}}\right)=\Pi(\gamma(\tau))=\mathbf{Y}+\tau \xi_{\mathbf{Y}}+o\left(\mathbf{1 1}^{\mathrm{T}} \tau^{2}\right)$ and concludes that $\left.\frac{d \mathbf{R}_{\mathbf{Y}}\left(\tau \xi_{\mathbf{Y}}\right)}{d \tau}\right|_{\tau=0}=\xi_{\mathbf{Y}}$.

\subsection{The Quotient Low-Rank Positive Multinomial Manifold}

As stated earlier, the considered problem exhibits nonisolated solutions. Indeed, given a solution $\mathbf{Y} \in \mathcal{M}_{p}^{n}$ and an orthogonal matrix $\mathbf{O} \in \mathcal{O}^{p}$, the point YO represent another solution. Define the relationship $\sim$ on $\mathcal{M}_{p}^{n}$ such that

$$
\mathbf{Y}_{1} \sim \mathbf{Y}_{2} \Leftrightarrow \exists \mathbf{O} \in \mathcal{O}^{p} \text { s.t. } \mathbf{Y}_{1} \mathbf{O}=\mathbf{Y}_{2}
$$

Clearly, the relationship $\sim$ defines an equivalence relationship. Let the set $\overline{\mathcal{M}}_{p}^{n}=\mathcal{M}_{p}^{n} / \sim$, or equivalently $\overline{\mathcal{M}}_{p}^{n}=\mathcal{M}_{p}^{n} / \mathcal{O}^{p}$, be the quotient manifold (see Appendix A for a formal proof that the set $\overline{\mathcal{M}}_{p}^{n}$ admits a manifold structure) of $\mathcal{M}_{p}^{n}$ by the above equivalence relationship. Points on $\overline{\mathcal{M}}_{p}^{n}$ are seen as equivalence classes denoted by $[\mathbf{Y}]=\overline{\mathbf{Y}}$ for $\mathbf{Y} \in \mathcal{M}_{p}^{n}$. Let $\pi: \mathcal{M}_{p}^{n} \rightarrow \overline{\mathcal{M}}_{p}^{n}$ be the canonical, or natural, projection of points to their equivalence class, i.e., $\pi(\mathbf{Y})=\overline{\mathbf{Y}}$.

Note that $f(\mathbf{Y})=g\left(\mathbf{Y} \mathbf{Y}^{\mathrm{T}}\right)$ is invariant under $\sim$ as $f\left(\mathbf{Y}_{1}\right)=f\left(\mathbf{Y}_{2}\right)$ for all $\mathbf{Y}_{1} \sim \mathbf{Y}_{2}$. Therefore, there exists a unique function $\bar{f}: \overline{\mathcal{M}}_{p}^{n} \rightarrow \mathbb{R}$, known as the projection of $f$, such that $f(\mathbf{Y})=\bar{f} \circ \pi(\mathbf{Y})$ for all $\mathbf{Y} \in \mathcal{M}_{p}^{n}$. The rest of this section is interested in studying the geometry of the quotient low-rank positive multinomial manifold in order to solve optimization problems of the form $\min _{\overline{\mathbf{Y}} \in \overline{\mathcal{M}}_{p}^{n}} \bar{f}(\overline{\mathbf{Y}})$. 
Let $\overline{\mathbf{Y}} \in \overline{\mathcal{M}}_{p}^{n}$, the equivalence class $\pi^{-1}(\overline{\mathbf{Y}})$ can be represented by the following set $\pi^{-1}(\overline{\mathbf{Y}})=\left\{\mathbf{Y O} \mid \mathbf{O} \in \mathcal{O}^{p}\right\}$. Recall that the vertical space $\mathcal{V}_{\mathbf{Y}} \mathcal{M}_{p}^{n}$ of $\overline{\mathbf{Y}}$ at $\mathbf{Y} \in \pi^{-1}(\overline{\mathbf{Y}})$ is given by $\mathcal{V}_{\mathbf{Y}} \mathcal{M}_{p}^{n}=\mathcal{T}_{\mathbf{Y}} \pi^{-1}(\overline{\mathbf{Y}})$. Let the function $F: \mathbb{R}^{n \times p} \rightarrow \mathbb{R}^{n \times n}$ defined by $F(\mathbf{Z})=\mathbf{Y} \mathbf{Y}^{\mathrm{T}}-\mathbf{Z Z}^{\mathrm{T}}$. Note that $\pi^{-1}(\mathbf{Y})$ is given by the level set of $F$ at $\mathbf{0}_{n \times n}$. Indeed, each $\mathbf{Z}$ satisfying $F(\mathbf{Z})=\mathbf{0}$ implies that $\mathbf{Y} \mathbf{Y}^{\mathrm{T}}=\mathbf{Z Z} \mathbf{Z}^{\mathrm{T}}$, i.e., $\mathbf{Z}=\mathbf{Y O}$ for some orthogonal matrix O. Furthermore, it is straightforward to conclude that $F$ is a constant-rank function from either the fact that $\mathbf{0}_{n \times n}$ is a regular value or by noting that $F$ is a submersion onto the set of positive matrices. Therefore, the tangent space at $\mathbf{Y}$ is given by the kernel of the indefinite directional derivative (see Lemma 1). In other words, the vertical space is given by

$$
\mathcal{V}_{\mathbf{Y}} \mathcal{M}_{p}^{n}=\mathcal{T}_{\mathbf{Y}} \pi^{-1}(\overline{\mathbf{Y}})=\left\{\xi_{\mathbf{Y}} \mid \xi_{\mathbf{Y}} \mathbf{Y}^{\mathrm{T}}+\mathbf{Y} \xi_{\mathbf{Y}}^{\mathrm{T}}=\mathbf{0}\right\} .
$$

Recall that $\mathbf{Y} \in \mathbb{R}_{*}^{n \times p}$ is a full rank matrix and define $\mathbf{Y}^{\perp}$ as any $n \times(n-p)$ matrix orthogonal complement of $\mathbf{Y}$ satisfying $\mathbf{Y}^{\mathrm{T}} \mathbf{Y}^{\perp}=\mathbf{0}$, then any matrix $\xi_{\mathbf{Y}}$ can be written as $\xi_{\mathbf{Y}}=\mathbf{Y} \mathbf{M}+\mathbf{Y}^{\perp} \mathbf{K}$ for some $p \times p$ matrix $\mathbf{M}$ and some $(n-p) \times p$ matrix $\mathbf{K}$. Hence, the characterization $\xi_{\mathbf{Y}} \mathbf{Y}^{\mathrm{T}}+\mathbf{Y} \xi_{\mathbf{Y}}^{\mathrm{T}}=\mathbf{0}$ of $\mathcal{V}_{\mathbf{Y}} \mathcal{M}_{p}^{n}$ can be rewritten as follows

$$
\mathbf{Y} \mathbf{M Y} \mathbf{Y}^{\mathrm{T}}+\mathbf{Y}^{\perp} \mathbf{K} \mathbf{Y}^{\mathrm{T}}+\mathbf{Y} \mathbf{M}^{\mathrm{T}} \mathbf{Y}^{\mathrm{T}}+\mathbf{Y} \mathbf{K}^{\mathrm{T}}\left(\mathbf{Y}^{\perp}\right)^{\mathrm{T}}=\mathbf{0} .
$$

Pre and post-multiplying the above equation by $\mathbf{Y}^{\mathrm{T}}$ and $\mathbf{Y}$, respectively gives $\left(\mathbf{Y}^{\mathrm{T}} \mathbf{Y}\right) \mathbf{M}\left(\mathbf{Y}^{\mathrm{T}} \mathbf{Y}\right)=$ $-\left(\mathbf{Y}^{\mathrm{T}} \mathbf{Y}\right) \mathbf{M}^{\mathrm{T}}\left(\mathbf{Y}^{\mathrm{T}} \mathbf{Y}\right)$. Since $\left(\mathbf{Y}^{\mathrm{T}} \mathbf{Y}\right)$ is invertible, this concludes that $\mathbf{M}$ is skew-symmetric, i.e., $\mathbf{M}+\mathbf{M}^{\mathrm{T}}=\mathbf{0}$. Similarly, pre and post-multiplying by $\left(\mathbf{Y}^{\perp}\right)^{\mathrm{T}}$ and $\mathbf{Y}^{\perp}$ allows to conclude that $\mathbf{K}=\mathbf{0}$. Therefore, we obtain the following alternate representation of the vertical space of $\mathcal{M}_{p}^{n}$ at $\mathbf{Y}$

$$
\mathcal{V}_{\mathbf{Y}} \mathcal{M}_{p}^{n}=\left\{\mathbf{Y M} \mid \mathbf{M} \in \mathcal{S}_{\text {skew }}^{p}\right\}
$$

Theorem 4. Consider the $\overline{\mathbf{Y}} \in \overline{\mathcal{M}}_{p}^{n}$. The bi-linear form defined on $\mathcal{T}_{\overline{\mathbf{Y}}} \overline{\mathcal{M}}_{p}^{n} \times \mathcal{T}_{\overline{\mathbf{Y}}} \overline{\mathcal{M}}_{p}^{n}$ by

$$
\left\langle\xi_{\overline{\mathbf{Y}}}, \eta_{\overline{\mathbf{Y}}}\right\rangle_{\overline{\mathbf{Y}}}=\operatorname{Tr}\left(\bar{\xi}_{\mathbf{Y}}^{\mathrm{T}} \bar{\eta}_{\mathbf{Y}}\right), \mathbf{Y} \in \pi^{-1}(\overline{\mathbf{Y}})
$$

is a well-defined Riemannian metric that is compatible with $\sim$ and turns $\overline{\mathcal{M}}_{p}^{n}$ into a Riemannian quotient manifold. The horizontal distribution of $\overline{\mathbf{Y}} \in \overline{\mathcal{M}}_{p}^{n}$ at $\mathbf{Y} \in \pi^{-1}(\overline{\mathbf{Y}})$ is

$$
\mathcal{H}_{\mathbf{Y}} \mathcal{M}_{p}^{n}=\left\{\eta_{\mathbf{Y}} \in \mathcal{T}_{\mathbf{Y}} \mathcal{M}_{p}^{n} \mid \eta_{\mathbf{Y}}^{\mathrm{T}} \mathbf{Y}=\mathbf{Y}^{\mathrm{T}} \eta_{\mathbf{Y}}\right\}
$$

Proof: As stated in Section 3, it is sufficient to show that the Riemannian metric on the embedding space is compatible with the equivalence relationship $\sim$. Hence, this theorem is established by expressing the horizontal lifts $\bar{\eta}_{\mathbf{Y}}$ of tangent vectors $\eta_{\overline{\mathbf{Y}}}$ as function of an arbitrary representative $\mathbf{Y} \in \pi^{-1}(\overline{\mathbf{Y}})$. Afterwards, it is shown that the inner product does not depend on the representative at which the horizontal lift is computed.

Recall that the horizontal distribution $\mathcal{H}_{\mathbf{Y}} \mathcal{M}_{p}^{n}$ is given by the orthogonal complement of the vertical space $\mathcal{V}_{\mathbf{Y}} \mathcal{M}_{p}^{n}$ in the tangent space $\mathcal{T}_{\mathbf{Y}} \mathcal{M}_{p}^{n}$. The orthogonal complement of the vertical space is parameterized by the following

$$
\begin{aligned}
& \mathcal{V}_{\mathbf{Y}}^{\perp} \mathcal{M}_{p}^{n}=\left\{\eta_{\mathbf{Y}} \in \mathbb{R}^{n \times p} \mid\left\langle\xi_{\mathbf{Y}}, \eta_{\mathbf{Y}}\right\rangle_{\mathbf{Y}}=0, \forall \xi_{\mathbf{Y}} \in \mathcal{V}_{\mathbf{Y}} \mathcal{M}_{p}^{n}\right\} \\
& \quad=\left\{\eta_{\mathbf{Y}} \in \mathbb{R}^{n \times p} \mid \operatorname{Tr}\left(\eta_{\mathbf{Y}}^{\mathrm{T}} \mathbf{Y} \mathbf{M}\right)=0, \forall \mathbf{M} \in \mathcal{S}_{\text {skew }}^{p}\right\} .
\end{aligned}
$$

The set of skew-symmetric matrices can be reparameterized as the difference of general matrices and its transpose, i.e., $\mathbf{M}=\mathbf{A}-\mathbf{A}^{\mathrm{T}}$ for some $\mathbf{A} \in \mathbb{R}^{p \times p}$. Therefore, the equality in (23) can be expressed as

$$
\operatorname{Tr}\left(\eta_{\mathbf{Y}}^{\mathrm{T}} \mathbf{Y} \mathbf{A}\right)=\operatorname{Tr}\left(\mathbf{Y}^{\mathrm{T}} \eta_{\mathbf{Y}} \mathbf{A}\right), \forall \mathbf{A} \in \mathbb{R}^{p \times p} .
$$

Therefore, we obtain $\eta_{\mathbf{Y}}^{\mathrm{T}} \mathbf{Y}=\mathbf{Y}^{\mathrm{T}} \eta_{\mathbf{Y}}$. Finally, the horizontal distribution is given by

$$
\begin{aligned}
\mathcal{H}_{\mathbf{Y}} \mathcal{M}_{p}^{n} & =\mathcal{V}_{\mathbf{Y}}^{\perp} \mathcal{M}_{p}^{n} \cap \mathcal{T}_{\mathbf{Y}} \mathcal{M}_{p}^{n} \\
& =\left\{\eta_{\mathbf{Y}} \in \mathcal{T}_{\mathbf{Y}} \mathcal{M}_{p}^{n} \mid \eta_{\mathbf{Y}}^{\mathrm{T}} \mathbf{Y}=\mathbf{Y}^{\mathrm{T}} \eta_{\mathbf{Y}}\right\}
\end{aligned}
$$

From the definition of the sets, it is clear that $\mathcal{H}_{\mathbf{Y}} \mathcal{M}_{p}^{n} \oplus$ $\mathcal{V}_{\mathbf{Y}} \mathcal{M}_{p}^{n} \subseteq \mathcal{T}_{\mathbf{Y}} \mathcal{M}_{p}^{n}$. Finally, note that $\operatorname{Dim}\left(\mathcal{V}_{\mathbf{Y}} \mathcal{M}_{p}^{n}\right)=$ $\frac{p(p-1)}{2}$ and $\operatorname{Dim}\left(\mathcal{H}_{\mathbf{Y}} \mathcal{M}_{p}^{n}\right)=n p-\frac{p(p-1)}{2}-n$ which gives $\mathcal{H}_{\mathbf{Y}} \mathcal{M}_{p}^{n} \oplus \mathcal{V}_{\mathbf{Y}} \mathcal{M}_{p}^{n}=\mathcal{T}_{\mathbf{Y}} \mathcal{M}_{p}^{n}$ as anticipated.

Let $\bar{\xi}_{\mathbf{Y}} \in \mathcal{H}_{\mathbf{Y}} \mathcal{M}_{p}^{n}$ represent the horizontal lift of $\xi_{\overline{\mathbf{Y}}} \in \mathcal{T}_{\overline{\mathbf{Y}}} \overline{\mathcal{M}}_{p}^{n}$ for $\mathbf{Y} \in \pi^{-1}(\overline{\mathbf{Y}})$. The following lemma characterizes the horizontal lift within the same equivalence class with respect to the horizontal lift of an arbitrary representative

Lemma 2. Let $\overline{\mathbf{Y}} \in \overline{\mathcal{M}}_{p}^{n}$ and $\mathbf{Y} \in \mathcal{M}_{p}^{n}$ such that $\mathbf{Y} \in$ $\pi^{-1}(\overline{\mathbf{Y}})$. For any $\mathbf{O} \in \mathcal{O}^{p}$, the following holds

$$
\bar{\xi}_{\mathbf{Y O}}=\bar{\xi}_{\mathbf{Y}} \mathbf{O} \text {. }
$$

Proof: Define $\mathcal{U}_{\mathbf{Y}}=\left\{[\mathbf{Z}] \mid \mathbf{Z Z}^{\mathrm{T}} \mathbf{1}=c \mathbf{1}, \mathbf{Z}^{\mathrm{T}} \mathbf{Y} \in \mathcal{O}^{p}, c \in\right.$ $\mathbb{R}\}$. To show that $\mathcal{U}_{\mathbf{Y}}$ is a manifold, consider the function $F_{c}$ defined by $F_{c}(\mathbf{Z})=\left[\mathbf{Z Z}^{\mathrm{T}} \mathbf{1}-c \mathbf{1}, \mathbf{Z}^{\mathrm{T}} \mathbf{Y} \mathbf{Y}^{\mathrm{T}} \mathbf{Z}-\mathbf{I}\right]^{\mathrm{T}}$. The set $\mathcal{U}_{\mathbf{Y}}^{c}=\left\{\mathbf{Z} \mid \mathbf{Z Z}^{\mathrm{T}} \mathbf{1}=c \mathbf{1}, \mathbf{Z}^{\mathrm{T}} \mathbf{Y} \in \mathcal{O}^{p}\right\}$ is the level set of $F$ at $\mathbf{0}$. Notice that the function $F$ is smooth and that it is a constant-rank function from the fact that $\mathbf{0}$ is a regular value. Hence, using the implicit function theorem, it is concluded that $\mathcal{U}_{\mathrm{Y}}^{c}$ is a manifold. As the union of disjoint, closed, and Hausdorff manifolds is a manifold, it can be concluded that $\mathcal{U}_{\mathbf{Y}}=\left\{\mathbf{Z} \mid \mathbf{Z Z}^{\mathrm{T}} \mathbf{1}=\right.$ $\left.c \mathbf{1}, \mathbf{Z}^{\mathrm{T}} \mathbf{Y} \in \mathcal{O}^{p}, c \in \mathbb{R}\right\}$ is a manifold. Finally, using similar steps as those provided in Appendix $\mathrm{A}$, the quotient set $\mathcal{U}_{\mathbf{Y}}=\left\{[\mathbf{Z}] \mid \mathbf{Z Z}^{\mathrm{T}} \mathbf{1}=c \mathbf{1}, \mathbf{Z}^{\mathrm{T}} \mathbf{Y} \in \mathcal{O}^{p}, c \in \mathbb{R}\right\}$ can be demonstrated to be a manifold. Let the function $\sigma_{\mathbf{Y}}: \mathcal{U}_{\mathbf{Y}} \rightarrow \mathbb{R}^{n \times p}$ be given by

$$
\sigma_{\mathbf{Y}}([\mathbf{Z}])=\mathbf{Z} \mathbf{Z}^{\mathrm{T}} \mathbf{Y} .
$$

Note that $\sigma_{\mathbf{Y}}([\mathbf{Z}])$ can be written as $\sigma_{\mathbf{Y}}([\mathbf{Z}])=\mathbf{Z O}$ for $\mathbf{O}=\mathbf{Z}^{\mathrm{T}} \mathbf{Y}$ being an orthogonal matrix. Therefore, $\sigma_{\mathbf{Y}}$ satisfies $\pi\left(\sigma_{\mathbf{Y}}([\mathbf{Z}])\right)=\pi(\mathbf{Z O})=[\mathbf{Z}]$, i.e., it represents a right inverse of the natural projection $\pi$. Therefore, the operator $\mathrm{D}\left(\pi\left(\sigma_{\mathbf{Y}}([\mathbf{Z}])\right)\right) \circ \mathrm{D}\left(\sigma_{\mathbf{Y}}([\mathbf{Z}])\right)$ represents the identity map on $\mathcal{T}_{[\mathbf{Z}]} \mathcal{U}_{\mathbf{Y}}$. Furthermore, let $\mathbf{O} \in \mathcal{O}^{p}$ and note that the function satisfies the additional property $\sigma_{\mathbf{Y O}}([\mathbf{Z}])=\mathbf{Z Z}^{\mathrm{T}} \mathbf{Y O}=\sigma_{\mathbf{Y}}([\mathbf{Z}]) \mathbf{O}$. Let $\mathcal{S}_{\mathbf{Y}}$ be the image of $\mathcal{U}_{\mathbf{Y}}$ by $\sigma_{\mathbf{Y}}$. The set $\mathcal{S}_{\mathbf{Y}}$ admits the following parameterization

$$
\mathcal{S}_{\mathbf{Y}}=\left\{\mathbf{A} \in \mathbb{R}^{n \times p} \mid \mathbf{A}=\mathbf{Z Z}^{\mathrm{T}} \mathbf{Y},[\mathbf{Z}] \in \mathcal{U}_{\mathbf{Y}}\right\} .
$$


The set $\mathcal{S}_{\mathbf{Y}}$ has the following explicit characterization as

$$
\begin{aligned}
\mathcal{S}_{\mathbf{Y}}=\left\{\mathbf{A} \in \mathbb{R}^{n \times p} \mid\left(\mathbf{A} \mathbf{Y}^{\mathrm{T}}+\mathbf{Y} \mathbf{A}^{\mathrm{T}}\right) \mathbf{1}\right. & =c \mathbf{1}, c \in \mathbb{R}, \\
\mathbf{A}^{\mathrm{T}} \mathbf{Y} & \left.=\mathbf{Y}^{\mathrm{T}} \mathbf{A}\right\} .
\end{aligned}
$$

Indeed, let $\mathbf{A}=\mathbf{Z Z}^{\mathrm{T}} \mathbf{Y}$, then we have

$$
\begin{aligned}
\left(\mathbf{A Y} \mathbf{Y}^{\mathrm{T}}+\mathbf{Y} \mathbf{A}^{\mathrm{T}}\right) \mathbf{1} & =\left(\mathbf{Z Z}^{\mathrm{T}} \mathbf{Y} \mathbf{Y}^{\mathrm{T}}+\mathbf{Y} \mathbf{Y}^{\mathrm{T}} \mathbf{Z} \mathbf{Z}^{\mathrm{T}}\right) \mathbf{1} \\
& \stackrel{(\mathrm{a})}{=} \mathbf{Z} \mathbf{Z}^{\mathrm{T}} \mathbf{1}+c \mathbf{1}=2 c \mathbf{1}=c^{\prime} \mathbf{1},
\end{aligned}
$$

with the equality in $(a)$ comes from the fact that $\mathbf{Y} \mathbf{Y}^{\mathrm{T}} \mathbf{1}=$ 1. Furthermore, we have $\mathbf{A}^{\mathrm{T}} \mathbf{Y}=\left(\mathbf{Z Z}^{\mathrm{T}} \mathbf{Y}\right)^{\mathrm{T}} \mathbf{Y}=$ $\mathbf{Y}^{\mathrm{T}} \mathbf{Z Z} \mathbf{Z}^{\mathrm{T}} \mathbf{Y}=\mathbf{Y}^{\mathrm{T}} \mathbf{A}$. The equalities above conclude that $\mathcal{S}_{\mathbf{Y}}$ is included in the set described in (25). Finally, note that both representations in (24) and (25) have the same dimension $n(p-1)-\frac{p(p-1)}{2}$ which gives that (25) is a valid parameterization of $\mathcal{S}_{\mathbf{Y}}$. Such parameterization allows to get the indefinite directionnal derivative of $\sigma_{\mathbf{Y}}([\mathbf{Z}])$ as follows

$$
\begin{aligned}
& \mathrm{D} \sigma_{\mathbf{Y}}([\mathbf{Z}])=\mathcal{T}_{\mathbf{A}} \mathcal{S}_{\mathbf{Y}} \\
& =\left\{\eta_{\mathbf{Y}} \in \mathbb{R}^{n \times p} \mid\left(\mathbf{Y} \eta_{\mathbf{Y}}^{\mathrm{T}}+\eta_{\mathbf{Y}} \mathbf{Y}^{\mathrm{T}}\right) \mathbf{1}=\mathbf{0}, \eta_{\mathbf{Y}}^{\mathrm{T}} \mathbf{Y}=\mathbf{Y}^{\mathrm{T}} \eta_{\mathbf{Y}}\right\} \\
& \quad=\left\{\eta_{\mathbf{Y}} \in \mathcal{T}_{\mathbf{Y}} \mathcal{M}_{p}^{n} \mid \eta_{\mathbf{Y}}^{\mathrm{T}} \mathbf{Y}=\mathbf{Y}^{\mathrm{T}} \eta_{\mathbf{Y}}\right\}=\mathcal{H}_{\mathbf{Y}} \mathcal{M}_{p}^{n},
\end{aligned}
$$

which concludes the following characterization of the horizontal lift $\bar{\xi}_{\mathbf{Y}}=\mathrm{D}\left(\sigma_{\mathbf{Y}}\right)([\mathbf{Z}])[\xi]$. Using the above equality combined with the previously derived property $\sigma_{\mathbf{Y O}}([\mathbf{Z}])=\sigma_{\mathbf{Y}}([\mathbf{Z}]) \mathbf{O}$, the horizontal lift over the equivalence class can be written as

$$
\begin{aligned}
\bar{\xi}_{\mathbf{Y O}} & =\mathrm{D}\left(\sigma_{\mathbf{Y O}}\right)([\mathbf{Z}])[\xi]=\mathrm{D}\left(\sigma_{\mathbf{Y}} \mathbf{O}\right)([\mathbf{Z}])[\xi] \\
& =\mathrm{D}\left(\sigma_{\mathbf{Y}}\right)([\mathbf{Z}])[\xi] \mathbf{O}+\sigma_{\mathbf{Y}} \mathrm{D}(\mathbf{O})([\mathbf{Z}])[\xi] \\
& \stackrel{(a)}{=} \bar{\xi}_{\mathbf{Y}} \mathbf{O},
\end{aligned}
$$

wherein the simplification (a) is obtained by noticing that the second term is zero as $\mathbf{O}$ is an arbitrary variable constant with respect to the directional derivative.

Using the result of Lemma 2, it becomes clear that the metric proposed in (22) is compatible with the equivalence relationship $\sim$. Indeed, for $\overline{\mathbf{Y}} \in \overline{\mathcal{M}}_{p}^{n}$ and any predecessor $\mathbf{Y}_{1}$ and $\mathbf{Y}_{2}=\mathbf{Y}_{1} \mathbf{O}$ in $\pi^{-1}(\overline{\mathbf{Y}})$, we have the following

$$
\begin{aligned}
\left\langle\xi_{\overline{\mathbf{Y}}}, \eta_{\overline{\mathbf{Y}}}\right\rangle_{\overline{\mathbf{Y}}} & =\operatorname{Tr}\left(\bar{\xi}_{\mathbf{Y}_{2}}^{\mathrm{T}} \bar{\eta}_{\mathbf{Y}_{2}}\right) \\
& =\operatorname{Tr}\left(\bar{\xi}_{\mathbf{Y}_{1} \mathbf{O}}^{\mathrm{T}} \bar{\eta}_{\mathbf{Y}_{1} \mathbf{O}}\right) \\
& =\operatorname{Tr}\left(\mathbf{O}^{T} \bar{\xi}_{\mathbf{Y}_{1}}^{\mathrm{T}} \bar{\eta}_{\mathbf{Y}_{1}} \mathbf{O}\right) \\
& =\operatorname{Tr}\left(\bar{\xi}_{\mathbf{Y}_{1}}^{\mathrm{T}} \bar{\eta}_{\mathbf{Y}_{1}}\right), \forall \xi_{\overline{\mathbf{Y}}}, \eta_{\overline{\mathbf{Y}}} \in \mathcal{T}_{\overline{\mathbf{Y}}} \overline{\mathcal{M}}_{p}^{n} .
\end{aligned}
$$

Therefore, the Riemannian metric is independent of the chosen representation which concludes that $\overline{\mathcal{M}}_{p}^{n}$ admits a Riemmanian manifold structure.

Let $\mathcal{P}_{\mathbf{Y}}^{\mathcal{V}}$ and $\mathcal{P}_{\mathbf{Y}}^{\mathcal{H}}$ be the orthogonal projections from the ambient space to the vertical space $\mathcal{V}_{\mathbf{Y}} \mathcal{M}_{p}^{n}$ and horizontal space $\mathcal{H}_{\mathbf{Y}} \mathcal{M}_{p}^{n}$, respectively. Furthermore, let $\mathcal{P}_{\mathbf{Y}}$ be the orthogonal projection from the ambient space to the tangent space $\mathcal{T}_{\mathbf{Y}} \mathcal{M}_{p}^{n}$. Recall that the ambient space can be decomposed as $\mathbb{R}^{n \times p}=\mathcal{T}_{\mathbf{Y}} \mathcal{M}_{p}^{n} \oplus \mathcal{T}_{\mathbf{Y}}^{\perp} \mathcal{M}_{p}^{n}$ wherein the tangent space can be expressed as $\mathcal{T}_{\mathbf{Y}} \mathcal{M}_{p}^{n}=\mathcal{V}_{\mathbf{Y}} \mathcal{M}_{p}^{n} \oplus$ $\mathcal{H}_{\mathbf{Y}} \mathcal{M}_{p}^{n}$. Therefore, $\mathcal{P}_{\mathbf{Y}}^{\mathcal{H}}(\mathbf{Z})$ can be written as $\mathcal{P}_{\mathbf{Y}}^{\mathcal{H}}\left(\mathcal{P}_{\mathbf{Y}}(\mathbf{Z})\right)$ which reduces the study of $\mathcal{P}_{\mathbf{Y}}^{\mathcal{H}}$ to the tangent space $\mathcal{T}_{\mathbf{Y}} \mathcal{M}_{p}^{n}$.
Given a tangent vector $\xi_{\mathbf{Y}} \in \mathcal{T}_{\mathbf{Y}} \mathcal{M}_{p}^{n}$, it can be decomposed into a vertical and horizontal components as $\xi_{\mathbf{Y}}=\mathcal{P}_{\mathbf{Y}}^{\mathcal{V}}\left(\xi_{\mathbf{Y}}\right)+\mathcal{P}_{\mathbf{Y}}^{\mathcal{H}}\left(\xi_{\mathbf{Y}}\right)$. Recall that $\mathcal{P}_{\mathbf{Y}}^{\mathcal{V}}\left(\xi_{\mathbf{Y}}\right)=\mathbf{Y} \mathbf{M}$ for some skew symmetric matrix $\mathbf{M}$ and $\mathcal{P}_{\mathbf{Y}}^{\mathcal{H}}\left(\xi_{\mathbf{Y}}\right)^{\mathrm{T}} \mathbf{Y}=$ $\mathbf{Y}^{\mathrm{T}} \mathcal{P}_{\mathbf{Y}}^{\mathcal{H}}\left(\xi_{\mathbf{Y}}\right)$. Therefore, matrix $\mathbf{M}$ satisfies the Sylvester equation $\left(\mathbf{Y}^{\mathrm{T}} \mathbf{Y}\right) \mathbf{M}+\mathbf{M}\left(\mathbf{Y}^{\mathrm{T}} \mathbf{Y}\right)=\mathbf{Y}^{\mathrm{T}} \mathcal{P}_{\mathbf{Y}}(\mathbf{Z})-\mathcal{P}_{\mathbf{Y}}(\mathbf{Z})^{\mathrm{T}} \mathbf{Y}$. Finally, rearranging the terms of the decomposition gives that the orthogonal projection of $\mathbf{Z}$ from the ambient space $\mathbb{R}^{n \times p}$ to the horizontal $\mathcal{H}_{\mathbf{Y}} \mathcal{M}_{p}^{n}$ is $\mathcal{P}_{\mathbf{Y}}^{\mathcal{H}}(\mathbf{Z})=$ $\mathcal{P}_{\mathbf{Y}}(\mathbf{Z})$ - YM where $\mathbf{M}$ is the solution to the above Sylvester equation. In other words, the quotient Riemannian gradient can be written as a function of the Euclidean and embedded Riemannian gradients as follows $\operatorname{grad} \bar{f}(\overline{\mathbf{Y}})=\mathcal{P}_{\mathbf{Y}}^{\mathcal{H}}(\operatorname{Grad} f(\mathbf{Y}))=\operatorname{grad} f(\mathbf{Y})-\mathbf{Y M}$,

with $\mathbf{M}$ being the solution to the Sylvester equation

$$
\left(\mathbf{Y}^{\mathrm{T}} \mathbf{Y}\right) \mathbf{M}+\mathbf{M}\left(\mathbf{Y}^{\mathrm{T}} \mathbf{Y}\right)=\mathbf{Y}^{\mathrm{T}} \operatorname{grad} f(\mathbf{Y})-\operatorname{grad} f(\mathbf{Y})^{\mathrm{T}} \mathbf{Y} .
$$

The expression of the Riemannian Hessian on the quotient space is obtained by projecting the directional derivative of the Riemannian gradient on the quotient manifold which gives

$$
\text { hess } \bar{f}(\overline{\mathbf{Y}})\left[\xi_{\overline{\mathbf{Y}}}\right]=\mathcal{P}_{\mathbf{Y}}^{\mathcal{H}}\left(\text { hess } f(\mathbf{Y})\left[\bar{\xi}_{\mathbf{Y}}\right]-\bar{\xi}_{\mathbf{Y}} \mathbf{M}-\mathbf{Y} \dot{\mathbf{M}}\right) \text {, }
$$

with $\mathbf{M}$ being the solution to (27) and $\dot{M}$ satisfying

$$
\begin{aligned}
& \left(\mathbf{Y}^{\mathrm{T}} \mathbf{Y}\right) \dot{\mathbf{M}}+\dot{\mathbf{M}}\left(\mathbf{Y}^{\mathrm{T}} \mathbf{Y}\right)=\bar{\xi}_{\mathbf{Y}}^{\mathrm{T}} \operatorname{grad} f(\mathbf{Y})-\operatorname{grad} f(\mathbf{Y})^{\mathrm{T}} \bar{\xi}_{\mathbf{Y}} \\
& \quad+\mathbf{Y}^{\mathrm{T}} \text { hess } f(\mathbf{Y})\left[\bar{\xi}_{\mathbf{Y}}\right]-\text { hess } f(\mathbf{Y})\left[\bar{\xi}_{\mathbf{Y}}\right]^{\mathrm{T}} \mathbf{Y} \\
& \quad-2\left(\bar{\xi}_{\mathbf{Y}}^{\mathrm{T}} \mathbf{Y} \mathbf{M}+\mathbf{M} \bar{\xi}_{\mathbf{Y}}^{\mathrm{T}} \mathbf{Y}\right) .
\end{aligned}
$$

Let the retraction $R_{Y}$ be defined on the tangent space $\mathcal{T}_{\mathbf{Y}} \mathcal{M}_{p}^{n}$ by $\mathbf{R}_{\mathbf{Y}}\left(\xi_{\mathbf{Y}}\right)=\Pi\left(\mathbf{Y}+\xi_{\mathbf{Y}}\right)$. The proof that the above operator represents a retraction on $\mathcal{M}_{p}^{n}$ is omitted as it mirrors the steps used in the proof of Theorem 3. Indeed, the proposed retraction represents the first order approximation of the retraction in (19). Finally, consider the retraction

$$
\overline{\mathrm{R}}_{\overline{\mathbf{Y}}}\left(\xi_{\overline{\mathbf{Y}}}\right)=\pi\left(\Pi\left(\mathbf{Y}+\bar{\xi}_{\mathbf{Y}}\right)\right)
$$

for $\mathbf{Y} \in \pi^{-1}(\overline{\mathbf{Y}})$. The aforementioned operator represents a well-defined function as it does not depend on the representative $\mathbf{Y} \in \pi^{-1}(\overline{\mathbf{Y}})$. Indeed, let $\overline{\mathbf{Y}} \in \overline{\mathcal{M}}_{p}^{n}$ and consider a couple of representatives $\mathbf{Y}$ and $\mathbf{Y O}$ in $\pi^{-1}(\overline{\mathbf{Y}})$. Let $\mathbf{D}$ be the diagonal matrix such that $\mathbf{D}\left(\mathbf{Y}+\bar{\xi}_{\mathbf{Y}}\right)\left(\mathbf{Y}+\bar{\xi}_{\mathbf{Y}}\right)^{\mathrm{T}} \mathbf{D}$ is doubly stochastic matrix, then

$$
\begin{aligned}
& \mathbf{D}\left(\mathbf{Y O}+\bar{\xi}_{\mathbf{Y O}}\right)\left(\mathbf{Y O}+\bar{\xi}_{\mathbf{Y O}}\right)^{\mathrm{T}} \mathbf{D} \\
& \quad=\mathbf{D}\left(\mathbf{Y O}+\bar{\xi}_{\mathbf{Y}} \mathbf{O}\right)\left(\mathbf{Y} \mathbf{O}+\bar{\xi}_{\mathbf{Y}} \mathbf{O}\right)^{\mathrm{T}} \mathbf{D} \\
& =\mathbf{D}\left(\mathbf{Y}+\bar{\xi}_{\mathbf{Y}}\right)\left(\mathbf{Y}+\bar{\xi}_{\mathbf{Y}}\right)^{\mathrm{T}} \mathbf{D} .
\end{aligned}
$$

In other words, $\pi\left(\Pi\left(\mathbf{Y O}+\bar{\xi}_{\mathbf{Y O}}\right)\right)=\pi\left(\Pi\left(\mathbf{Y}+\bar{\xi}_{\mathbf{Y}}\right)\right)$ which shows that $\bar{R}_{\bar{Y}}$ satisfies (11). Therefore, we conclude that $R_{Y}$ is a well-defined retraction on the quotient manifold.

\section{Optimization Algorithms on the Posi- TIVE MULTINOMIAL MANIFOLD}

This section exploits the previously studied embedded and quotient low-rank positive multinomial manifolds 


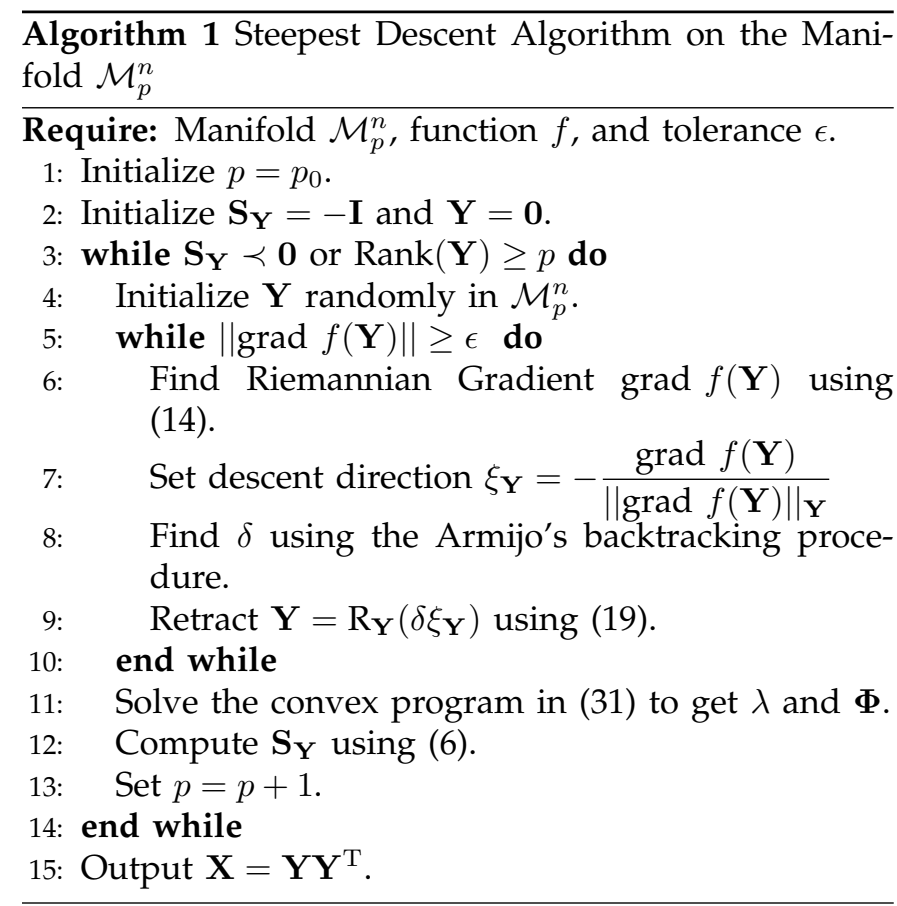

to propose a first and a second-order optimization algorithms on these manifolds. The optimality conditions derived in Section 2 are then utilized to solve the original optimization problem (1) by means of solving its nonconvex reformulation (2). In the remaining of the paper, we assume that the optimization problem (1) is convex and well-defined, in the sense that its optimal solution $\mathbf{X}^{*}$ is rank deficient. In other words, there exists a $p \leq n$ such that the optimal solution to (2) with dimension $p$ satisfies the optimality conditions in Section 2.

For simplicity, this section focuses only on the steepest descent algorithm and Newton's method as a first and second-order approaches on the embedded and quotient manifolds, respectively. The simulation section uses the conjugate gradient and the trust region algorithms for their enhanced performance.

This manuscript suggests solving a sequence of optimization problems with increasing dimension $p$, i.e., an increasing rank of the solution, until the theoretical guarantees in Theorem 1 are satisfied. Recall that the condition that $\mathbf{S}_{\mathbf{Y}}$ being positive semidefinite is sufficient to guarantee the optimality of $\mathbf{X}=\mathbf{Y Y}^{\mathrm{T}}$ for a convex objective function. Therefore, initializing $p$ with some $p_{0}$ (usually $p_{0}=1$ ), the algorithm solve the optimization problem (2) using the Riemannian geometry tool developed in Section 4. In particular, starting at a random point $\mathbf{Y}$ in the manifold, the Euclidean gradient is projected onto the tangent space to obtain a descent direction $\xi_{\mathbf{Y}}$. For enhanced performance, the step size $\delta$ is optimized through the Armijo's backtracking procedure [17]. Afterwards, the intermediate point on the tangent space is retracted to the manifold. The process is repeated until the gradient vanishes. After obtaining the solution $\mathbf{Y}^{*}$, the dual variable $\boldsymbol{\Phi}$ is obtained by solving

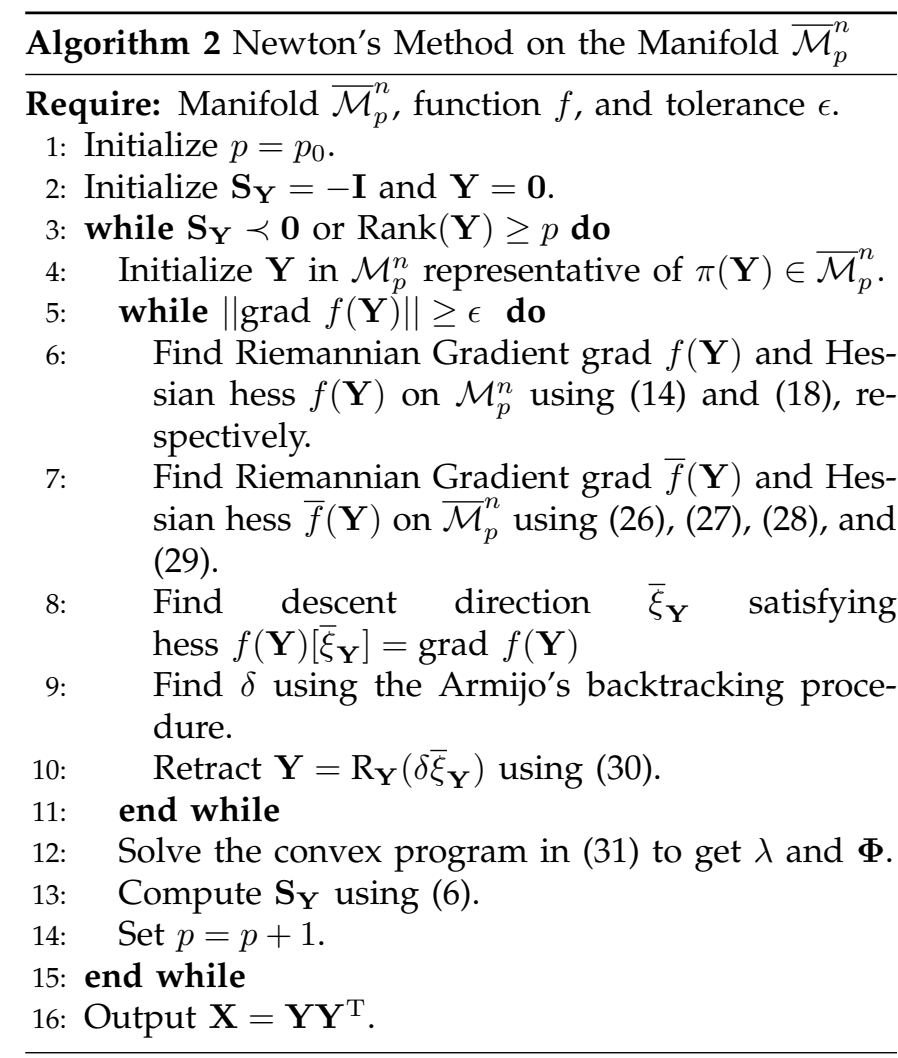

the convex problem

$$
\begin{aligned}
\min _{\mathbf{\Phi} \in \mathcal{S}^{n}} & \left\|\left(\operatorname{Grad} f(\mathbf{Y})+\lambda(\boldsymbol{\Phi}) \mathbf{1}^{T}+\mathbf{1} \lambda(\boldsymbol{\Phi})^{T}-\mathbf{\Phi}\right) \mathbf{Y}\right\|_{F}^{2} \\
\text { s.t. } & \mathbf{\Phi} \geq \mathbf{0} \\
& \mathbf{Y} \mathbf{Y}^{T} \odot \mathbf{\Phi}=\mathbf{0}
\end{aligned}
$$

with $\lambda(\boldsymbol{\Phi})=\frac{1}{n}\left(\mathbf{I}-\frac{1}{2 n} \mathbf{1 1}^{T}\right)(\boldsymbol{\Phi}-\operatorname{Grad} f(\mathbf{Y})) \mathbf{1}$. Given the unique solution $\Phi$, the second dual variable $\lambda$ is obtained by the expression above which allows to compute matrix $\mathbf{S}_{\mathbf{Y}}$ and check its non-negativity. In case of success, the optimal $\mathbf{X}^{*}=\mathbf{Y}^{*}\left(\mathbf{Y}^{*}\right)^{\mathrm{T}}$ is returned. Otherwise, the process is repeated for an increased value of $p$. For second order differential functions, the convergence can be attested by checking that the solution is rank (almost) deficient from Theorem 2 which is cheaper than checking the first order conditions in Theorem 1. In other words, under the above assumption, one does not need to solve the convex program (31) and to derive $\mathbf{S}_{\mathbf{Y}}$. The steps of the algorithm are summarized in Algorithm 1.

Newton's method on the quotient manifold follows similar steps as the one in on embedded manifolds by properly substituting the geometrical objects. The algorithm sequentially increases the rank of the solution and solve the optimization problem using Newton's method on the manifold $\overline{\mathcal{M}}_{p}^{n}$. The Newton's step on the quotient manifold is accomplished by computing the Riemannian gradient and Hessian and finding the tangent vector $\xi_{\mathbf{Y}}$ satisfying hess $f(\mathbf{Y})\left[\bar{\xi}_{\mathbf{Y}}\right]=\operatorname{grad} f(\mathbf{Y})$. Afterwards, the step size $\delta$ is computed, and the point retracted onto the manifold. In a similar manner as before, the algorithm stops for the first rank $p$ for which the matrix $\mathbf{S}_{\mathbf{Y}}$ is non- 
negative or $\operatorname{Rank}(\mathbf{Y})<p$ for second order differentiable functions. The steps are summarized in Algorithm 2.

\section{Numerical Results}

This section extensively investigates the performance of the proposed manifold-based algorithms in Section 5. Subsection 6.1 exploits the proposed framework to provide recovery of a similarity clustering, also known as affinity in the clustering literature, via convex programming. Although multiple methods can be utilized to solve convex programs, this manuscript only considers the convex solver CVX [39] for its simplicity, genericness, and widespread in the community. This paper uses realworld data obtained through crowdsourcing on Amazon Mechanical Turk [40]. Subsection 6.2 performs a similar comparison for a large dimension and a varying number of clusters. Due to logistics issues that arise from the considered high dimension, Subsection 6.2 uses synthetic data generated from a stochastic block model which approximates well the real-world data. Subsection 6.3 of the simulation emphasizes the performance of Algorithm 1 and Algorithm 2 in finding the optimal solution for an unknown rank $p$.

Note that even though the second order method on the embedded manifold is not guaranteed to converge to an extreme point due to the existence of non-isolated solutions, such behavior is not observed in our experiments. Therefore, this part compares the performance of the proposed conjugate gradient (CG) and the trust region (TR) methods on both the embedded and the quotient manifold. Unlike the steepest descent, the conjugate gradient algorithm requires a vector transport. However, the design of such an operator, e.g., using [41], [42], falls outside the scope of this manuscript. In these simulations, the default ManOpt vector transport, i.e., the orthogonal projection onto the tangent space, has been used to obtain the proposed figures. The performance of the proposed algorithms is tested against the generic convex solver CVX [39] and the conjugate gradient algorithm on the symmetric multinomial $\mathcal{M}^{n}=$ $\left\{\mathbf{X} \in \mathcal{S}^{n} \mid \mathbf{X} \succ \mathbf{0}, \mathbf{X}>\mathbf{0}, \mathbf{X} \mathbf{1}=\mathbf{1}\right\}$. The CG on $\mathcal{M}^{n}$ can be easily obtained using the first and second-order geometries of the manifold derived in [30]. Furthermore, the CG method on $\mathcal{M}^{n}$ is readily available in the Matlab toolbox ManOpt [43]. In Subsections 6.2 and 6.3, the problems are evaluated over a large number of iterations, and the mean value is presented. All simulations are carried out using the Matlab toolbox ManOpt [43] on an Intel Xeon Processor E5-1650 v4 (15M cache, $3.60 \mathrm{GHz})$ computer with $32 \mathrm{~Gb} 2.4 \mathrm{GHz}$ DDR4 RAM.

\subsection{Similarity Clustering via Convex Programming}

This part suggests retrieving the cluster structure of an affinity matrix obtained from crowdsourcing on Amazon Mechanical Turk. Images of $n=473$ dogs from the Standford Dogs Dataset [44] of $p=3$ different breeds,
TABLE 2

\section{Performance of the Proposed Methods for Clustering}

\begin{tabular}{|c|c|c|c|}
\hline Algorithm & Running Time & Var. of Inf. & Err. Rate \\
\hline \hline CVX & $3183.060 \mathrm{~s}$ & 0.5404 & $6.3 \%$ \\
\hline ALM & $2.848651 \mathrm{~s}$ & 0.8688 & $12.68 \%$ \\
\hline CG on $\mathcal{M}^{n}$ & $6.121646 \mathrm{~s}$ & 0.5543 & $6.7 \%$ \\
\hline CG on $\mathcal{M}_{p}^{n}$ & $4.777171 \mathrm{~s}$ & 0.5403 & $6.3 \%$ \\
\hline CG on $\overline{\mathcal{M}}_{p}^{n}$ & 3.813541 & 0.5501 & $6.5 \%$ \\
\hline
\end{tabular}

i.e., Norfolk Terrier (172 images), Toy Poodle (151 images) and Bouvier des Flandres (150 images), are used in the experiment. At each trial, non-expert workers are required to determine if the two dogs presented on the screen have the same breed or not. Each worker is given a set of 30 images, and around 600 responses have been collected. Out of the total possible edges $\frac{n(n-1)}{2}=111628$, only 17260 edges, i.e., around $15 \%$ of the total number of entries, are queried and used to construct the adjancacy matrix $\mathbf{A}$. The $(i, j)$ th entries of $\mathbf{A}$ is defined as the average binary response from the various workers to the question "Are dogs $i$ and $j$ from the same breed?" Out of these 17260 queried edges, 3941 responses are wrong which gives a $22 \%$ error rate. Without errors, matrix $\mathbf{A}$ would be of rank $p=3$. However, due to errors, this part reveals the cluster structure of $\mathbf{A}$ by solving the following convex optimization problem whose theoretical guarantees are studied in [12]

$$
\min _{\mathbf{X} \in \mathcal{M}^{n}} \frac{1}{2}\|\mathbf{A}-\mathbf{X}\|_{F}^{2}+\lambda \operatorname{Tr}(\mathbf{X}),
$$

wherein the restricted Frobenius $\bar{F}$ norm is computed only over the observed entries of $\mathbf{A}$. The optimization problem is solved using the numerical optimization toolbox CVX [39], a specialized approximate and fast algorithm [45], known as augmented Lagrange multipliers (ALM), and the conjugate gradient algorithm on symmetric multinomial. Afterwards, the same problem is solved by reformulating $\mathbf{X}=\mathbf{Y} \mathbf{Y}^{\mathrm{T}}$ and using our proposed methods on the embedded and the quotient manifold.

The quality of the recovery is attested through the computation of the variation of information [46] between the reached cluster structure and the ground truth. Table 2 illustrates the running time and the performance of the above-mentioned optimization methods. To be fair in comparison, only the conjugate gradient algorithm is employed over the different manifolds. Clearly, the proposed methods considerably outperform the generic solver CVX and the CG algorithm on the Riemannian symmetric multinomial in term of running time while maintaining the quality of the solution. The simulation also shows that the quotient manifold provides better results than its embedded counterpart which is expected as the quotient manifold reduces the dimension of the ambient space by grouping all equivalent solutions. 


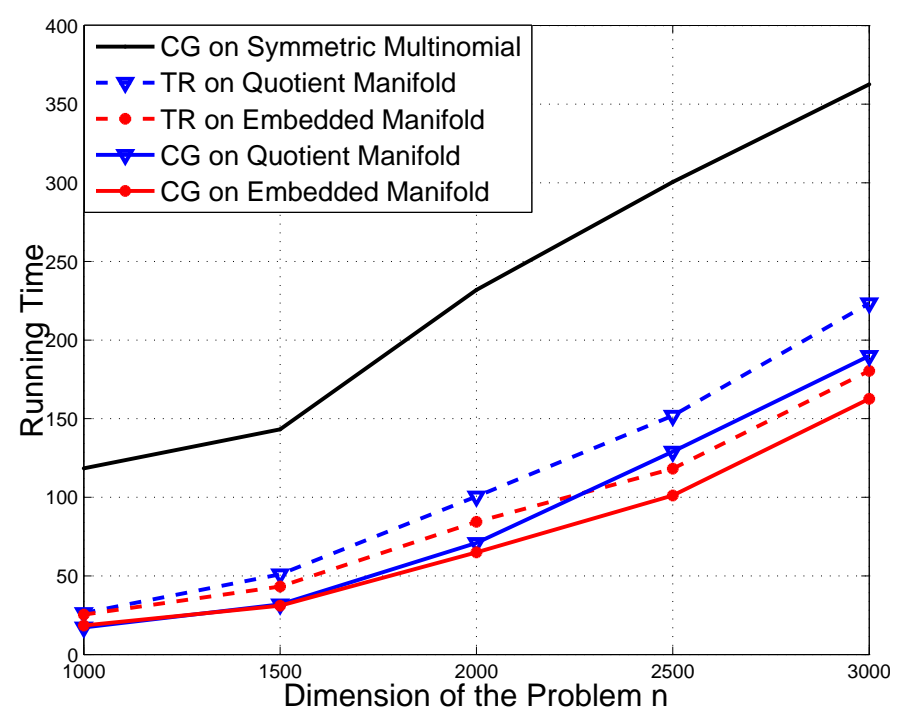

Fig. 1. Performance of the proposed optimization scheme in clustering in terms of running time against the system dimension $n$ for a number of clusters $p=4 n / 1000$.

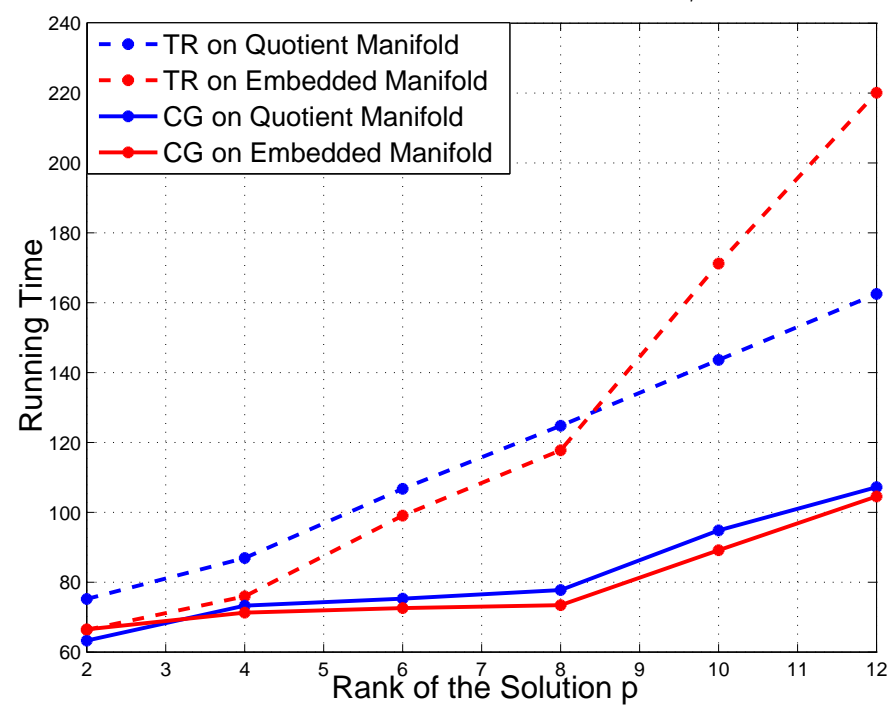

Fig. 2. Performance of the proposed Riemannian optimization algorithm against the number of clusters $p$ for a system of dimension $n=2000$.

\subsection{High Dimension Community Detection}

This part proposes solving the clustering problem for a large number of entries $n$, e.g., large number of dogs in Subsection 6.1, using synthetic data. In particular, the crowdsourcing part is simulated by sampling from a stochastic block model similar to the one studied in [12] to obtain the similarity matrix. The number of clusters, e.g., the number of breeds of dogs in Subsection 6.1, is also variable so as to study multiple scenarios. The size of clusters is chosen randomly from a set of predefined sizes for each dimension such that the recovery is theoretically guaranteed. Furthermore, the parameters of the stochastic block model are selected so that the theoretical guarantees proposed in [12] are valid which is further confirmed by an almost null total variation of information between the ground truth and the reached solution. The first part of these simulations compares the time performance of the proposed first and second order methods on the embedded and quotient manifolds against the performance achieved by a firstorder method on the symmetric multinomial $\mathcal{M}^{n}$. The second part shows the performance of the proposed solution against a system of huge dimension. For such large dimension, neither the generic CVX nor the specialized Riemannian symmetric multinomial are applicable. The final part plots the running time of the suggested methods against the number of clusters $p$ for a fixed dimension $n$.

TABLE 3

Running time of the proposed methods in a system with large dimension for a number of clusters $p=n / 1000$.

\begin{tabular}{|c|c|c|c|}
\hline \multirow{2}{*}{ Algorithm } & \multicolumn{3}{|c|}{ Dimension of the Problem } \\
\cline { 2 - 4 } & 8000 & 9000 & 10000 \\
\hline \hline TR on $\mathcal{M}_{p}^{n}$ & 2462 & 3240 & 4147 \\
\hline TR on $\overline{\mathcal{M}}_{p}^{n}$ & 2375 & 3145 & 4147 \\
\hline CG on $\mathcal{M}_{p}^{n}$ & 1605 & 2627 & 3721 \\
\hline CG on $\overline{\mathcal{M}}_{p}^{n}$ & 1660 & 2731 & 3765 \\
\hline
\end{tabular}

Figure 1 plots the running time of the proposed first and second order methods again the dimension of the problem $n$ for clusters scaling as $p=4 n / 1000$. As a base of comparison, this section plots the performance of the conjugate gradient algorithm on the symmetric multinomial. The running time of the second order method on the symmetric multinomial requires solving an $n \times n$ system of linear equations which largely deteriorate its performance and thus is omitted in these simulations. Figure 1 clearly displays that the proposed methods achieve the same performance with drastically lower running time. The behavior is further illustrated in Table 3 wherein the system dimension is very large $8000 \leq$ $n \leq 10000$ for a number of clusters $p=n / 1000$. The configuration of Figure 1 is prohibitively complex to run either CVX or the symmetric multinomial. Nevertheless, our proposed methods achieve the optimal solution in reasonable running time.

Figure 2 plots the performance of the proposed algorithms in clustering large data sets versus the number of clusters. As shown in the analysis in the manuscript, the dimension of the suggested manifold increases with the rank $p$, i.e., number of clusters. Such fact is attested by Figure 2. The figure especially displays that the second order method is more sensitive to a change in $p$ as a $p \times p$ linear system needs to be solved at each iteration for these second order methods.

\subsection{Optimization with Unknown Rank $p$}

This final set of simulation attests the performance of Algorithm 1 and Algorithm 2 in finding the optimal 


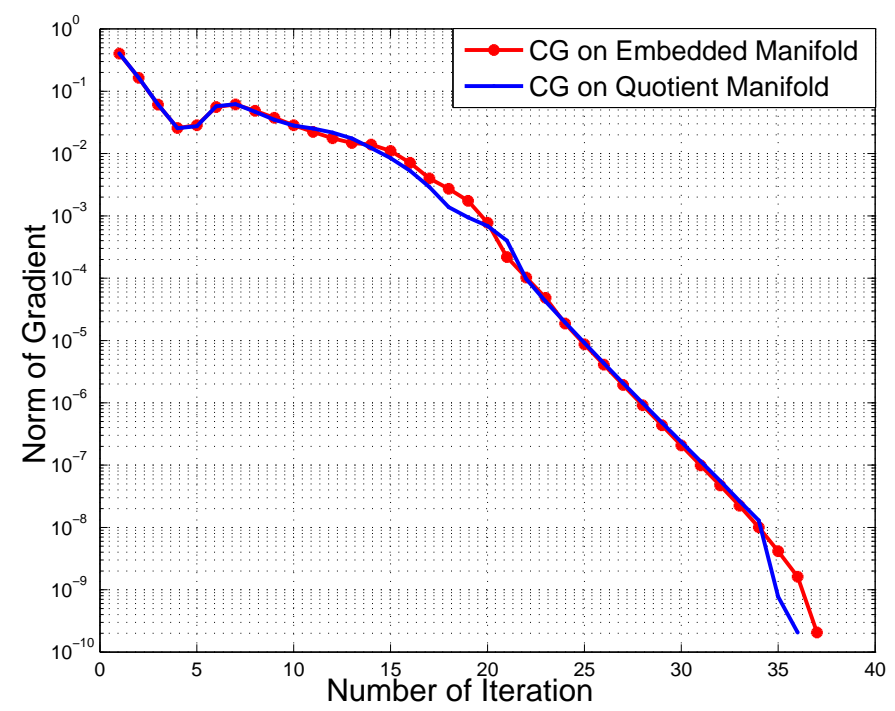

Fig. 3. Convergence rate of the conjugate gradient algorithm on the embedded $\mathcal{M}_{p}^{n}$ and quotient manifold $\overline{\mathcal{M}}_{p}^{n}$ for $n=2000$ and $p=10$.

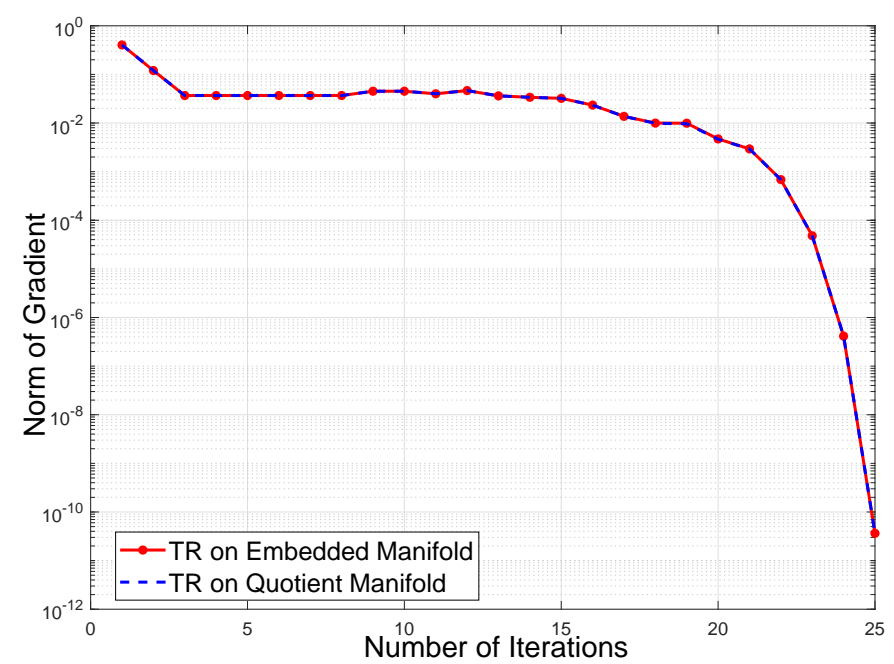

Fig. 4. Convergence rate of the trust-region algorithm on the embedded $\mathcal{M}_{p}^{n}$ and quotient manifold $\overline{\mathcal{M}}_{p}^{n}$ for $n=$ 2000 and $p=10$.

solution for an unknown rank $p$. A matrix $\mathbf{A}$ is generated from a low-rank $p$ doubly stochastic matrix and corrupted with a zero mean white Gaussian noise with unitary total power. This part solves the following denoising problem $\min _{\mathbf{Y} \in \mathcal{M}_{p}^{n}}\left\|\mathbf{A}-\mathbf{Y} \mathbf{Y}^{\mathrm{T}}\right\|_{F}^{2}$. Assuming the solution rank $p$ is known, the first part of the simulation confirms the linear and quadratic convergence behavior of the proposed first and second order methods, respectively. The second part runs Algorithm 1 and Algorithm 2 in order to find the optimal solution without any prior knowledge of the rank. Both algorithms are initiated with rank $p_{0}=2$ which is increased until the convergence criteria of Algorithm 1 and Algorithm 2 are reached. The convergence is attested by checking that the solution is rank (almost) deficient from Theorem 2 which is cheaper than checking the first order conditions in Theorem 1.

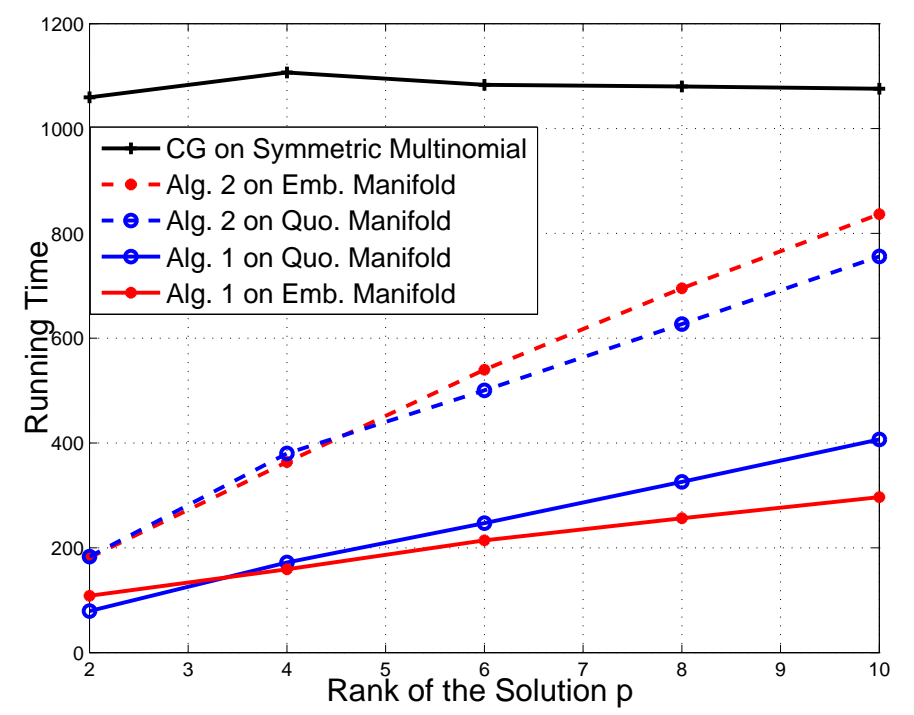

Fig. 5. Performance of Algorithm 1 and Algorithm 2 in finding the optimal solution without prior knowledge of the rank against the number of clusters $p$ in a system of dimension $n=2000$.

Figure 3 illustrates the convergence rate of the conjugate gradient method (first-order method) on the proposed embedded and quotient manifolds. The figure reveals that the proposed first-order methods exhibit a linear behavior as anticipated. One can note that the linear response of these methods mimics the one of unconstrained optimization as the Riemannian method transforms a constrained optimization into an unconstrained one. Similarly, Figure 4 plots the convergence rate of the second-order methods. These second order methods achieve a superlinear convergence as compared with the first order methods. The figure also illustrates that the quadratic behavior is reached after a certain number of iterations which can be explained by the fact that the quadratic approximation of the objective function in the trust region method is tight only in a region close to the optimal value.

Figure 5 shows the running time of the proposed algorithms in solving the above optimization problem with unknown solution rank $p$. Recall that the symmetric multinomial solves the problem over the $\frac{n(n-1)}{2}$ space, unlike our method that reduces the dimension to $n(p-1)$. However, the geometry of the embedded and quotient manifold is more complex than the one of the symmetric multinomial. Furthermore, as the rank of the solution is unknown, Algorithm 1 and Algorithm 2 solve the optimization multiple times with increasing rank. Nevertheless, Figure 5 attests that for a reasonable number of clusters $p$, our proposed Algorithm 1 and Algorithm 2 both perform better than the symmetric multinomial.

\section{Conclusion}

This manuscript designs efficient optimization algorithms for solving optimization problems on the set of 
positive semidefinite stochastic matrices. Assuming that the optimal solution has a much lower rank than the ambient dimension, the paper reformulates the problem by introducing the factorization of the optimization variable $\mathbf{X}=\mathbf{Y Y}^{\mathrm{T}}$. Theoretical guarantees under which the reparametrized problem produces satisfactory solution are derived. The paper introduced an embedded and a quotient Riemannian manifolds in order to solve the reparameterized problem. The efficiency of the proposed framework is attested using both real-world and synthetic data.

\section{ACKNOWLEDGMENTS}

The authors would like to express their gratitude to the reviewers for their careful reading, their insightful, and valuable comments which helped to highly improve the quality of the manuscript.

\section{REFERENCES}

[1] A. Douik and B. Hassibi, "Low-rank Riemannian optimization on positive semidefinite stochastic matrices with applications to graph clustering," in International Conference on Machine Learning, 2018, pp. 1298-1307.

[2] Z. Yang and E. Oja, "Unified development of multiplicative algorithms for linear and quadratic nonnegative matrix factorization," IEEE Transactions on Neural Networks, vol. 22, no. 12, pp. 18781891, Dec 2011.

[3] C. H. Ding, T. Li, and M. I. Jordan, "Convex and semi-nonnegative matrix factorizations," IEEE transactions on pattern analysis and machine intelligence, vol. 32, no. 1, pp. 45-55, 2010.

[4] V. Chandrasekaran, B. Recht, P. A. Parrilo, and A. S. Willsky, "The convex geometry of linear inverse problems," Foundations of Computational mathematics, vol. 12, no. 6, pp. 805-849, 2012.

[5] B. Vandereycken, "Low-rank matrix completion by riemannian optimization," SIAM Journal on Optimization, vol. 23, no. 2, pp. 1214-1236, 2013.

[6] A. Douik and B. Hassibi, "An improved initialization for lowrank matrix completion based on rank-l updates," in 2018 IEEE International Conference on Acoustics, Speech and Signal Processing (ICASSP). IEEE, 2018, pp. 3959-3963.

[7] R. A. Brualdi, "Some applications of doubly stochastic matrices," Linear Algebra and its Applications, vol. 107, pp. 77-100, 1988.

[8] K. Shanmugam, A. G. Dimakis, and M. Langberg, "Local graph coloring and index coding," in IEEE International Symposium on Information Theory (ISIT' 2013), Istanbul, Turkey. IEEE, 2013, pp. $1152-1156$.

[9] M. Mehlum, "Doubly stochastic matrices and the assignment problem," Master's thesis, University of Oslo, 2012.

[10] D. Hartfiel and J. Spellmann, "A role for doubly stochastic matrices in graph theory," Proceedings of the American Mathematical Society, vol. 36, no. 2, pp. 389-394, 1972.

[11] R. Arora, M. Gupta, A. Kapila, and M. Fazel, "Clustering by left-stochastic matrix factorization," in Proceedings of the 28th International Conference on Machine Learning (ICML'11), 2011, pp. 761-768.

[12] R. K. Vinayak and B. Hassibi, "Similarity clustering in the presence of outliers: Exact recovery via convex program," in 2016 IEEE International Symposium on Information Theory (ISIT' 2016), July 2016, pp. 91-95.

[13] Z. Yang and E. Oja, "Clustering by low-rank doubly stochastic matrix decomposition," in Proceedings of the 29th International Coference on International Conference on Machine Learning, ser. ICML'12, USA, 2012, pp. 707-714.

[14] X. Wang, F. Nie, and H. Huang, "Structured doubly stochastic matrix for graph based clustering: Structured doubly stochastic matrix," in Proc. of the 22Nd ACM SIGKDD International Conference on Knowledge Discovery and Data Mining, ser. KDD '16, 2016, pp. $1245-1254$.
[15] A. Douik and B. Hassibi, "A riemannian approach for graphbased clustering by doubly stochastic matrices," in 2018 IEEE Statistical Signal Processing Workshop (SSP). IEEE, 2018, pp. 806810.

[16] R. Zass and A. Shashua, "Doubly stochastic normalization for spectral clustering," in NIPS, 2006, pp. 1569-1576.

[17] P.-A. Absil, R. Mahony, and R. Sepulchre, Optimization Algorithms on Matrix Manifolds. Princeton, NJ: Princeton University Press, 2008.

[18] S. Homer and M. Peinado, "Design and performance of parallel and distributed approximation algorithms for maxcut," Journal of Parallel and Distributed Computing, vol. 46, no. 1, pp. 48-61, 1997.

[19] C. Helmberg and F. Rendl, "A spectral bundle method for semidefinite programming," SIAM Journal on Optimization, vol. 10, no. 3, pp. 673-696, 2000.

[20] S. Burer and R. D. Monteiro, "A nonlinear programming algorithm for solving semidefinite programs via low-rank factorization," Mathematical Programming, vol. 95, no. 2, pp. 329-357, 2003.

[21] D. G. Luenberger, "The gradient projection method along geodesics," Management Science, vol. 18, no. 11, pp. 620-631, 1972.

[22] D. Gabay, "Minimizing a differentiable function over a differential manifold," Journal of Optimization Theory and Applications, vol. 37, no. 2, pp. 177-219, 1982.

[23] K. Huper and J. Trumpf, "Newton-like methods for numerical optimization on manifolds," in Proc. of 38th Asilomar Conference on Signals, Systems and Computers, 2004, Pacific Grove, CA, USA, vol. 1, Nov 2004, pp. 136-139 Vol.1.

[24] C. G. Baker, P.-A. Absil, and K. A. Gallivan, "An implicit trustregion method on Riemannian manifolds," IMA J. Numer. Anal., vol. 28 , no. 4, pp. 665-689, 2008.

[25] P.-A. Absil, C. G. Baker, and K. A. Gallivan, "Trust-region methods on Riemannian manifolds," Foundations of Computational Mathematics, vol. 7, no. 3, pp. 303-330, 2007.

[26] W. Ring and B. Wirth, "Optimization methods on Riemannian manifolds and their application to shape space," SIAM Journal on Optimization, vol. 22, no. 2, pp. 596-627, 2012.

[27] I. Grubišić and R. Pietersz, "Efficient rank reduction of correlation matrices," Linear algebra and its applications, vol. 422, no. 2-3, pp. 629-653, 2007.

[28] S. Bonnabel and R. Sepulchre, "Riemannian metric and geometric mean for positive semidefinite matrices of fixed rank," SIAM Journal on Matrix Analysis and Applications, vol. 31, no. 3, pp. 10551070, 2009.

[29] M. Journée, F. Bach, P.-A. Absil, and R. Sepulchre, "Low-rank optimization on the cone of positive semidefinite matrices," SIAM Journal on Optimization, vol. 20, no. 5, pp. 2327-2351, 2010.

[30] A. Douik and B. Hassibi, "Manifold optimization over the set of doubly stochastic matrices: A second-order geometry," IEEE Transactions on Signal Processing, vol. 67, no. 22, pp. 5761-5774, 2019.

[31] M. Berger and B. Gostiaux, Differential geometry: manifolds, curves, and surfaces, ser. Graduate texts in mathematics. Springer-Verlag, 1988.

[32] J. Lee, Introduction to Topological Manifolds, ser. Graduate Texts in Mathematics. Springer New York, 2010.

[33] P. Petersen, Riemannian Geometry, ser. Graduate texts in mathematics. Springer, 1998.

[34] S. Boyd and L. Vandenberghe, Convex Optimization. New York, NY, USA: Cambridge University Press, 2004.

[35] J. Kyparisis, "On uniqueness of kuhn-tucker multipliers in nonlinear programming," Mathematical Programming, vol. 32, no. 2, pp. 242-246, 1985.

[36] C. M. Bishop, Neural Networks for Pattern Recognition. New York, NY, USA: Oxford University Press, Inc., 1995.

[37] J. Csima and B. Datta, "The DAD theorem for symmetric nonnegative matrices," Journal of Combinatorial Theory, Series A, vol. 12, no. 1, pp. 147 - 152, 1972.

[38] R. Sinkhorn, "A relationship between arbitrary positive matrices and doubly stochastic matrices," The annals of mathematical statistics, vol. 35, no. 2, pp. 876-879, 1964.

[39] M. Grant and S. Boyd, "CVX: Matlab software for disciplined convex programming, version 2.1," http://cvxr.com/cvx, Mar. 2014.

[40] M. Buhrmester, T. Kwang, and S. D. Gosling, "Amazon's mechanical turk: A new source of inexpensive, yet high-quality, data?" Perspectives on psychological science, vol. 6, no. 1, pp. 3-5, 2011. 
[41] H. Sato and T. Iwai, "A new, globally convergent riemannian conjugate gradient method," Optimization, vol. 64, no. 4, pp. 1011$1031,2015$.

[42] H. Sato, "A dai-yuan-type riemannian conjugate gradient method with the weak wolfe conditions," Computational Optimization and Applications, vol. 64, no. 1, pp. 101-118, 2016.

[43] N. Boumal, B. Mishra, P.-A. Absil, and R. Sepulchre, "Manopt, a Matlab toolbox for optimization on manifolds," Journal of Machine Learning Research, vol. 15, pp. 1455-1459, 2014. [Online]. Available: http:/ / www.manopt.org

[44] A. Khosla, N. Jayadevaprakash, B. Yao, and F.-F. Li, "Novel dataset for fine-grained image categorization: Stanford dogs," in Proc. CVPR Workshop on Fine-Grained Visual Categorization (FGVC), vol. 2, 2011, p. 1.

[45] Z. Lin, M. Chen, and Y. Ma, "The augmented lagrange multiplier method for exact recovery of corrupted low-rank matrices," arXiv preprint arXiv:1009.5055, 2010.

[46] M. Meilă, "Comparing clusterings by the variation of information," in Learning theory and kernel machines. Springer, 2003, pp. 173-187.

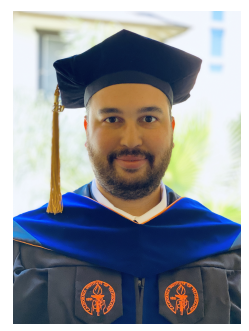

Ahmed Douik (S'13 M'20) received the Eng. degree in electronic and communication engineering (with first class honors) from the Ecole Polytechnique de Tunisie, Tunisia, in 2013, the M.S. degree in electrical engineering from King Abdullah University of Science and Technology, Thuwal, Saudi Arabia, in 2015, and the Ph.D. degree from the California Institute of Technology, Pasadena, CA, USA in 2020. His research interests include optimization, non-Convex methods, network coding, and cooperative communication. machine learning, cloud-radio access networks,

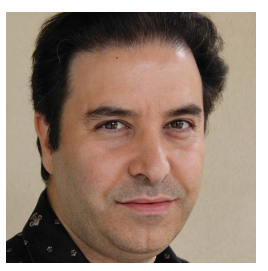

Babak Hassibi was born in Tehran, Iran, in 1967. He received the B.S. degree from the University of Tehran in 1989, and the M.S. and Ph.D. degrees from Stanford University in 1993 and 1996, respectively, all in electrical engineering.

He has been with the California Institute of Technology since January 2001, where he is currently the Mose and Lilian S. Bohn Professor of Electrical Engineering. From 20013-2016 he was the Gordon M. Binder/Amgen Professor of Electrical Engineering and from 2008-2015 he was Executive Officer of Electrical Engineering, as well as Associate Director of Information Science and Technology. From October 1996 to October 1998 he was a research associate at the Information Systems Laboratory, Stanford University, and from November 1998 to December 2000 he was a Member of the Technical Staff in the Mathematical Sciences Research Center at Bell Laboratories, Murray Hill, NJ. He has also held shortterm appointments at Ricoh California Research Center, the Indian Institute of Science, and Linkoping University, Sweden. His research interests include communications and information theory, control and network science, and signal processing and machine learning. He is the coauthor of the books (both with A.H. Sayed and T. Kailath) Indefinite Quadratic Estimation and Control: A Unified Approach to $H^{2}$ and $H^{\infty}$ Theories (New York: SIAM, 1999) and Linear Estimation (Englewood Cliffs, NJ: Prentice Hall, 2000). He is a recipient of an Alborz Foundation Fellowship, the 1999 O. Hugo Schuck best paper award of the American Automatic Control Council (with H. Hindi and S.P. Boyd), the 2002 National ScienceFoundation Career Award, the 2002 Okawa Foundation Research Grant for Information and Telecommunications, the 2003 David and Lucille Packard Fellowship for Science and Engineering, the 2003 Presidential Early Career Award for Scientists and Engineers (PECASE), and the 2009 Al-Marai Award for Innovative Research in Communications, and was a participant in the 2004 National Academy of Engineering "Frontiers in Engineering" program. 


\section{APPENDIX A \\ MANIfold Structure of $\overline{\mathcal{M}}_{p}^{n}$}

In order to show that the set $\overline{\mathcal{M}}_{p}^{n}=\mathcal{M}_{p}^{n} / \sim$ admits a manifold structure, it is sufficient to show that $\sim$ is regular [17], meaning that it satisfies the three following properties

1) $\operatorname{graph}(\sim)$ is an embedded submanifold of the product $\mathcal{M}_{p}^{n} \times \mathcal{M}_{p}^{n}$.

2) The projection $\pi_{1}: \operatorname{graph}(\sim) \rightarrow \mathcal{M}_{p}^{n}$ given by $\pi_{1}\left(\mathbf{Y}_{1}, \mathbf{Y}_{2}\right)=\mathbf{Y}_{1}$ is a submersion.

3) $\operatorname{graph}(\sim)$ is closed, wherein $\operatorname{graph}(\sim)=\left\{\left(\mathbf{Y}_{1}, \mathbf{Y}_{2}\right) \in \mathcal{M}_{p}^{n} \times \mathcal{M}_{p}^{n} \mid \mathbf{Y}_{1} \sim \mathbf{Y}_{2}\right\}$.

The proof that $\operatorname{graph}(\sim)$ is an embedded submanifold of the product $\mathcal{M}_{p}^{n} \times \mathcal{M}_{p}^{n}$ is omitted herein as it mirrors the steps in proving the same result for the Grassmann manifold. Indeed, note that $\operatorname{graph}(\sim) \subset \operatorname{graph}\left(\sim^{\prime}\right)$ with $\mathbf{Y}_{1} \sim^{\prime} \mathbf{Y}_{2}$ means that $\mathbf{Y}_{1} \mathbf{M}=\mathbf{Y}_{2}$ for some invertible $p \times p$ matrix $\mathbf{M}$. Therefore, $\operatorname{graph}(\sim)$ is an embedded submanifold as wanted.

For a matrix $\mathbf{Y} \in \mathcal{M}_{p}^{n}$, let $\mathbf{X} \in \mathbb{R}^{n \times p}$ satisfy $\mathbf{Y}+$ $t \mathbf{X} \in \mathcal{M}_{p}^{n}$ for $t$ in some interval $I$ containing zero. The existence of $\mathbf{X}$ is guaranteed by the fact that $\mathcal{M}_{p}^{n}$ represents an open set, and thus it contains an open ball centered at each $\mathbf{Y}$. For an orthogonal matrix $\mathbf{O} \in \mathcal{O}^{p}$, define the curve $\gamma(t)$ by $\gamma(t)=(\mathbf{Y}+t \mathbf{X},(\mathbf{Y}+t \mathbf{X}) \mathbf{O})$. For $t \in I$, it is clear that $\gamma(t) \in \operatorname{graph}(\sim)$. Furthermore, $\gamma(t)$ satisfies the property

Therefore, $\pi_{1}$ is a submersion.

$$
\left.\frac{d \pi_{1}(\gamma(t))}{d t}\right|_{t=0}=\left.\frac{d(\mathbf{Y}+t \mathbf{X})}{d t}\right|_{t=0}=\mathbf{X}
$$

Finally, the closure of $\operatorname{graph}(\sim)$ can be obtained by noticing that $\operatorname{graph}(\sim)$ is the pre-image of $\mathbf{0}_{n \times n}$ by the function $\left(\mathbf{Y}_{1}, \mathbf{Y}_{2}\right) \mapsto \mathbf{Y}_{1} \mathbf{Y}_{1}^{\mathrm{T}}-\mathbf{Y}_{2} \mathbf{Y}_{2}^{\mathrm{T}}$. Indeed, note that $\mathbf{Y}_{1} \mathbf{Y}_{1}^{\mathrm{T}}=\mathbf{Y}_{2} \mathbf{Y}_{2}^{\mathrm{T}}$ implies that $\mathbf{Y}_{1} \mathbf{O}=\mathbf{Y}_{2}$ for some orthogonal matrix $\mathbf{O} \in \mathcal{O}^{p}$. Given that the function is continuous and that $\left\{\mathbf{0}_{n \times n}\right\}$ is a closed set, we conclude that $\operatorname{graph}(\sim)$ is closed.

Combining the three results above allows concluding that $\overline{\mathcal{M}}_{p}^{n}$ admits a unique manifold structure known as the quotient manifold of $\mathcal{M}_{p}^{n}$ by $\sim$. However, note that the proof does not allow to conclude that $\overline{\mathcal{M}}_{p}^{n}$ inherits the Riemannian structure of $\mathcal{M}_{p}^{n}$ as it requires the Riemannian metric to be compatible with $\sim$ which is shown in Section 4. 